\title{
Galactic halo size in the light of recent AMS-02 data
}

\author{
N. Weinrich ${ }^{1}$, M. Boudaud ${ }^{2, \star}$, L. Derome ${ }^{1}$, Y. Génolini ${ }^{3}$, J. Lavalle ${ }^{4}$, D. Maurin ${ }^{1}$, P. Salati ${ }^{5}$, \\ P. Serpico ${ }^{5}$, and G. Weymann-Despres ${ }^{1}$
}

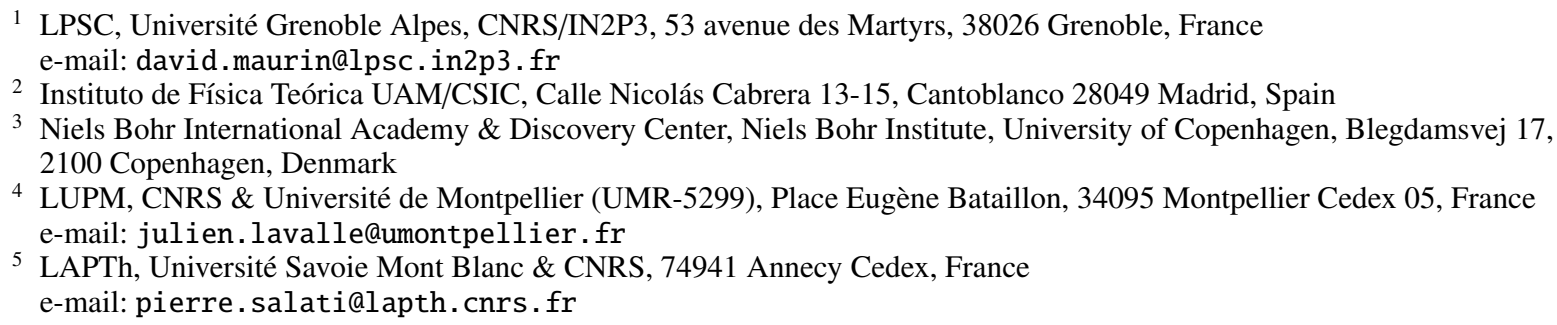

Received 1 April 2020 / Accepted 14 May 2020

\begin{abstract}
Context. The vertical diffusive halo size of the Galaxy, $L$, is a key parameter for dark matter indirect searches. It can be better determined thanks to recent AMS-02 data.

Aims. We set constraints on $L$ from $\mathrm{Be} / \mathrm{B}$ and ${ }^{10} \mathrm{Be} / \mathrm{Be}$ data, and we performed a consistency check with positron data. We detail the dependence of $\mathrm{Be} / \mathrm{B}$ and ${ }^{10} \mathrm{Be} / \mathrm{Be}$ on $L$ and forecast on which energy range better data would be helpful for future $L$ improvements. Methods. We used USINE v3.5 for the propagation of nuclei, and $\mathrm{e}^{+}$were calculated with the pinching method.

Results. The current AMS-02 Be/B ( 3\% precision) and ACE-CRIS ${ }^{10} \mathrm{Be} / \mathrm{Be}(\sim 10 \%$ precision) data bring similar and consistent constraints on $L$. The AMS- $02 \mathrm{Be} / \mathrm{B}$ data alone constrain $L=5_{-2}^{+3} \mathrm{kpc}$ at a $68 \%$ confidence level (spanning different benchmark transport configurations), a range for which most models do not overproduce positrons. Future experiments need to deliver percent-level accuracy on ${ }^{10} \mathrm{Be} /{ }^{9} \mathrm{Be}$ anywhere below $10 \mathrm{GV}$ to further constrain $L$.

Conclusions. Forthcoming AMS-02, HELIX, and PAMELA ${ }^{10} \mathrm{Be} /{ }^{9} \mathrm{Be}$ results will further test and possibly tighten the limits derived here. Elemental ratios involving radioactive species with different lifetimes (e.g. $\mathrm{Al} / \mathrm{Mg}$ and $\mathrm{Cl} / \mathrm{Ar}$ ) are also awaited to provide complementary and robuster constraints.
\end{abstract}

Key words. astroparticle physics - cosmic rays - Galaxy: halo

\section{Introduction}

Finding the Galactic cosmic-ray (CR) sources, solving the details of CR transport in the Galaxy, and using the CR as a channel to identify the nature of dark matter (DM) are among the main challenges in CR physics. In the last decade, antimatter data received a lot of scrutiny, owing to the interpretation of the positron fraction rise as a DM signal (Adriani et al. 2009; Bergström et al. 2008; Feng \& Zhang 2018), although standard astrophysics explanations are more likely (e.g. Hooper et al. 2009; Delahaye et al. 2010; Serpico 2012; Manconi et al. 2019). Similarly, the presence of a DM contribution in the $\bar{p}$ data is debated in the literature (Reinert \& Winkler 2018; Cholis et al. 2019; Cuoco et al. 2019; Boudaud et al. 2020). The latter channel is actually one of the best at constraining weakly interacting massive particles DM candidates in the $\mathrm{GeV}-\mathrm{TeV}$ mass range (e.g. Conrad \& Reimer 2017).

DM interpretations for antimatter CRs depend on both the transport and geometry parameters (Donato et al. 2004; Delahaye et al. 2008; Aramaki et al. 2016); the latter is mostly determined via $\mathrm{CR}$ radioactive clocks. These clocks have a lifetime of approximately a million years, one order of magnitude shorter than the typical CR propagation time in the Galaxy. Ratios of a secondary

$\star$ Deceased. (i.e. produced at the propagation stage only) unstable species to their stable counterpart, for instance ${ }^{10} \mathrm{Be} /{ }^{9} \mathrm{Be}$, allow one to break the degeneracy between the diffusion coefficient normalisation and the halo size of the Galaxy (e.g. Donato et al. 2002). However, CR isotopic separation in experiments is challenging. The first measurements were carried out more than forty years ago for ${ }^{10} \mathrm{Be}$ (Webber et al. 1973), ${ }^{36} \mathrm{Cl}$ (Young et al. 1981), ${ }^{26} \mathrm{Al}$ (Webber 1982), and ${ }^{54} \mathrm{Mn}$ (Webber et al. 1979); however, even recent measures (Connell 1998; Yanasak et al. 2001; Hams et al. 2004) are restricted to low energy mostly, that is, below a few hundreds of $\mathrm{MeV} / \mathrm{n}$. As an alternative and complementary approach, Webber \& Soutoul (1998) proposed to use elemental ratios (e.g. $\mathrm{Be} / \mathrm{B}, \mathrm{Al} / \mathrm{Mg}$ ) in which the CR clock appears both in the numerator (decayed fraction) and denominator (daughter fed by decaying CR). Elemental ratios have been measured up to hundreds of $\mathrm{GeV} / \mathrm{n}$, hence covering an energy range in which ${ }^{10} \mathrm{Be}$ goes from mostly decayed to meta-stable, with respect to the propagation time, at high energy. While waiting for the AMS-02 future release of Be isotope data, the high-precision $\mathrm{Be} / \mathrm{B}$ ratio already gives useful constraints (Evoli et al. 2020). However, the isotopic fraction of ${ }^{10} \mathrm{Be}$ in $\mathrm{B}$ is a few percent only, and the sensitivity of $\mathrm{Be} / \mathrm{B}$ to $L$ is partly drowned by the dominant presence of the stable nucleus ${ }^{7} \mathrm{Be}$ (Tomassetti 2015a).

The positron data have also been recently shown to provide interesting constraints on $L$ (Lavalle et al. 2014). In particular, 
for small $L$, the unavoidable secondary production, mostly from $\mathrm{H}$ and $\mathrm{He} \mathrm{CRs}$ on the interstellar medium (ISM), may overshoot low-energy data points. Therefore they can be used as a complementary probe to set a lower limit on $L$.

This study follows up on our previous effort to determine CR transport parameters (Génolini et al. 2019; Weinrich et al. 2020). We performed a joint analysis of $\mathrm{Li} / \mathrm{C}, \mathrm{B} / \mathrm{C}$, and various combinations of ${ }^{10} \mathrm{Be}$ data to determine $L$. We then drew models from the allowed regions of their parameter space and further checked their consistency with the positron constraint. We stress that in this analysis, as is the case in almost all similar studies, the halo size of the Galaxy is set to be a hard boundary where the CR density goes to zero. This is an effective modelling of a more realistic picture that would probably involve a rapidly growing diffusion coefficient in the halo (Di Bernardo et al. 2013; Tomassetti 2015b). In fact, a modelling of CR, gas, and wave interactions from first principles leads to the picture of a dynamic halo (Breitschwerdt et al. 1991; Zirakashvili et al. 1996). Recent results from Evoli et al. (2018) show, in particular, that the turbulent cascade from CR sources and the selfgeneration of waves by CRs can introduce an effective halo size. Full numerical simulations aimed at accurately computing CRs in a magneto-hydrodynamic framework will probably give more insight into this problem, as envisaged in Girichidis et al. (2020). In the meantime, the concept of hard boundary remains a useful benchmark for many CR-related studies.

The paper is organised as follows: in Sect. 2, we recall the propagation model and configurations used for the analysis. In Sect. 3, we assess the capability of ${ }^{10} \mathrm{Be} /{ }^{9} \mathrm{Be}, \mathrm{Be} / \mathrm{B}$, or $\mathrm{B} / \mathrm{C}$ current data to determine $L$, accounting for various modelling uncertainties; we then provide the resulting constraints on $L$. In Sect. 4, we discuss other possible observables to determine $L$, and we look at the flux of secondary positrons, given the $\mathrm{Be} / \mathrm{B}$ constraint on $L$. In Sect. 5, we summarise and conclude. Further details of the analysis and additional cross-checks are reported to the appendices: Appendix A provides scaling relations of the transport parameters with $L$ for stable secondary species - they extend, for all transport parameters, those that were given in Appendix C of Génolini et al. (2019); Appendix B highlights the constraints on $L$ set by various ${ }^{10} \mathrm{Be} /{ }^{9} \mathrm{Be}$ datasets (low-energy experiments, ISOMAX, and preliminary PAMELA analysis); Appendix $\mathrm{C}$ outlines why ${ }^{7} \mathrm{Be} /\left({ }^{9} \mathrm{Be}+{ }^{10} \mathrm{Be}\right)$, the most favourable isotopic ratio to extract experimentally, is of no practical use to constrain $L$.

\section{Model and configurations (BIG, SLIM, QUAINT)}

The details of the transport equation and approach we follow are detailed in Génolini et al. (2019) and in the companion paper (Weinrich et al. 2020). Here, we only recall the most important features of the model.

We assume the CR density to obey a steady-state diffusionadvection equation. The geometry of the diffusion halo defines the region in which CRs propagate and are confined, here considered an infinite slab of half-thickness $L$, the parameter we aim at determining in this analysis. In this geometry, CR sources and the gas are pinched in an infinitely thin plan (half-thickness $h=100 \mathrm{pc} \ll L$ ), where interactions with the gas (spallation, energy gains and losses) are restricted to. A convection term $V_{\mathrm{c}}$ is taken to be constant and perpendicular to the disc. We assume isotropic and homogeneous diffusion, and account for diffusion in momentum space: we follow the treatment of Seo et al. (1994), that is $K_{p p}(R, x) \propto 2 h \delta(z)\left(V_{\mathrm{A}} p\right)^{2} / K(R)$ where $V_{\mathrm{A}}$ is the Alfvénic speed of the plasma wave. These assump-
Table 1. Free $(\checkmark)$ and fixed parameters for the benchmark configurations analysed in this study, see Eq. (1).

\begin{tabular}{cccc}
\hline \hline Parameters & BIG & SLIM & QUAINT \\
\hline \multicolumn{5}{c}{ Low-rigidity } & parameters \\
$\eta$ & 1 & 1 & $\checkmark$ \\
$\delta_{1}$ & $\checkmark$ & $\checkmark$ & n/a \\
$s_{1}$ & 0.05 & 0.05 & n/a \\
$R_{1}$ & $\checkmark$ & $\checkmark$ & n/a \\
$V_{\mathrm{A}}$ & $\checkmark$ & n/a & $\checkmark$ \\
\hline Intermediate-rigidity parameters \\
$V_{\mathrm{c}}$ & $\checkmark$ & n/a & $\checkmark$ \\
$K_{0}$ & $\checkmark$ & $\checkmark$ & $\checkmark$ \\
$\delta$ & $\checkmark$ & $\checkmark$ & $\checkmark$ \\
\hline \multicolumn{5}{c}{ High-rigidity } & parameters \\
$\Delta_{\mathrm{h}}$ & 0.18 & 0.19 & 0.17 \\
$R_{\mathrm{h}}[\mathrm{GV}]$ & 247 & 237 & 270 \\
$s_{\mathrm{h}}$ & 0.04 & 0.04 & 0.04 \\
\hline \multicolumn{5}{c}{ Geometry parameter } \\
$L$ & $\checkmark$ & $\checkmark$ & $\checkmark$ \\
\hline \multicolumn{4}{c}{}
\end{tabular}

Notes. We schematically separate the parameters in several rigidity domains, but $V_{\mathrm{A}}$ and $V_{\mathrm{c}}$ impact fluxes across both the low- and intermediate-rigidity domain.

tions allow one to derive solutions semi-analytically (Jones et al. 2001; Maurin et al. 2001). Our calculations are performed with the code USINE V3.5 (Maurin 2020) ${ }^{1}$. For more details, we refer the reader to Génolini et al. (2019) and Weinrich et al. (2020).

Several theoretical studies have hinted at the possible presence of breaks at low- (Ptuskin et al. 2006) or high-rigidity (Blasi et al. 2012; Evoli et al. 2018). Actually, spectral breaks are seen in CR data at low- (Stone et al. 2013) and high-rigidity (Aguilar et al. 2018), and they can be connected to the presence of breaks in the diffusion coefficient at rigidities $R_{l} \approx 4-5 \mathrm{GV}$ (Génolini et al. 2019; Vittino et al. 2019; Weinrich et al. 2020) and $R_{h} \approx 250 \mathrm{GV}$ (Génolini et al. 2017, 2019; Reinert \& Winkler 2018; Evoli et al. 2019). For these reasons we take

$K(R)=\beta^{\eta} K_{0}\left\{1+\left(\frac{R}{R_{1}}\right)^{\frac{\delta_{1}-\delta}{s_{1}}}\right\}^{s_{1}}\left\{\frac{R}{1 \mathrm{GV}}\right\}^{\delta}\left\{1+\left(\frac{R}{R_{\mathrm{h}}}\right)^{\frac{\delta-\delta_{\mathrm{h}}}{s_{\mathrm{h}}}}\right\}^{-s_{\mathrm{h}}}$

In this study, we fix several parameters whose impact on the results is negligible: the three high-rigidity break parameters $\left(R_{h}, \delta_{h}, s_{h}\right)$ are set to the values reported in Génolini et al. (2019); the smoothness of the low-rigidity break parameter is fixed at $s_{l}=0.04$ (fast transition).

We use three benchmark configurations BIG, SLIM, and QUAINT defined in Génolini et al. (2019), whose relevant parameters are collected in Table 1 . In this analysis, BIG has 7 free parameters $\left(K_{0}, \delta, R_{l}, \delta_{l}, V_{\mathrm{c}}, V_{\mathrm{A}}, L\right)$. The configuration SLIM is a special case of BIG, with $V_{\mathrm{A}}=V_{\mathrm{c}}=0$ and $\eta=$ 1 , and it has 5 free parameters $\left(K_{0}, \delta, R_{l}, \delta_{l}, L\right)$. The configuration QUAINT is also a special case of BIG with no lowrigidity break, and it has 6 free parameters $\left(K_{0}, \delta, \eta, V_{\mathrm{c}}, V_{\mathrm{A}}, L\right)$. QUAINT is also an extension (because of the high-rigidity break) of older benchmark convection and reacceleration models used for instance in Maurin et al. (2010) and Di Bernardo et al. (2010).

1 https://lpsc.in2p3.fr/usine 
We fitted the model to the data via a $\chi^{2}$ minimisation,

$\chi^{2}=\sum_{t}\left(\sum_{q_{t}}\left(\mathcal{D}_{\mathrm{cov}}^{t, q_{t}}+\mathcal{N}_{\text {Sol.Mod. }}^{t, q_{t}}\right)\right)+\sum_{r} \mathcal{N}_{\mathrm{XS}}^{r}$,

where $t$ and $q_{t}$ run over the several flux ratios (e.g. Li/C, Be/C, $\mathrm{B} / \mathrm{C}$ ) measured at different periods, whereas $r$ runs over crosssection reactions. The non-diagonal $\mathcal{D}_{\text {cov }}^{t, q_{t}}$ term allows for $i j$ energy bins correlations in the data (covariance matrix). The $\mathcal{N}_{\text {Sol.Mod. }}^{t, q}$ and $\mathcal{N}_{\mathrm{Xs}}$ terms account for Solar modulation and crosssection nuisance parameters, respectively. More details on this procedure are given in Appendix B of Derome et al. (2019). For further details related to the $\mathrm{Li} / \mathrm{C}, \mathrm{Be} / \mathrm{C}$, and $\mathrm{B} / \mathrm{C}$ data uncertainties (correlation matrix of systematics), and also solar modulation and cross-section priors, we refer the reader to our companion paper (Weinrich et al. 2020).

The minimisation is performed with the MINUIT package (James \& Roos 1975), its MINOS algorithm also providing accurate (asymmetric) error bars even if the problem is very nonlinear. In practice, the MINUIT routines are directly called from USINE (Maurin 2020). We also carry out $O(100)$ minimisations from different starting points to ensure the true minimum is found (Weinrich et al. 2020). All uncertainties reported on the halo size in this study are derived from the profile likelihood method (with MINOS) at the $68 \%$ confidence level. As the $\chi^{2}$ definition accounts for energy correlations in the data uncertainties and nuisance parameters, the halo size uncertainties also account for them. We stress that uncertainties were derived on $\log (L)$ and are mostly symmetric on this parameter. For this reason, we loosely use the notation $1 \sigma$ in the following, and for instance, $2 \sigma$ limits can be estimated assuming a log-normal distribution for $L$.

\section{Halo size $L$ from $C R$ clocks}

Radioactive secondary species whose lifetime is shorter than escape time decay before experiencing the boundary of the Galaxy. These species are only sensitive to the diffusion coefficient $K$, whereas stable secondary species can escape and are sensitive to $K / L$. Any fit combining the information of a stable and radioactive secondary species breaks the $K / L$ degeneracy, allowing for the determination of $L$ (Donato et al. 2002).

Below, we focus on ${ }^{10} \mathrm{Be}\left(t_{1 / 2}=1.387 \mathrm{Myr}\right)$ and related ratios $\left({ }^{10} \mathrm{Be} /{ }^{9} \mathrm{Be},{ }^{10} \mathrm{Be} / \mathrm{Be}\right.$, and $\left.\mathrm{Be} / \mathrm{B}\right)$. To date, ${ }^{10} \mathrm{Be}$ is the only available $\mathrm{CR}$ chronometer for which high-precision data exist for the associated elemental flux, $\mathrm{Be}$. The $\mathrm{Al}, \mathrm{Cl}$, and $\mathrm{Mn}$ fluxes have not been released by the AMS-02 collaboration, yet.

\subsection{CR datasets and modulation levels}

This analysis is based on several datasets. Each dataset is associated to a solar modulation level depending on its data taking period. We use here the simple force-field approximation (Gleeson \& Axford 1967, 1968; Caballero-Lopez \& Moraal 2004), whose single parameter, $\phi_{\mathrm{FF}}$, is taken as a nuisance parameter in the analyses. As detailed in Weinrich et al. (2020), the solar modulation level (prior) for each dataset is based on the analysis of neutron monitor data (Maurin et al. 2015; Ghelfi et al. 2016, 2017a,b).

As in Weinrich et al. (2020), the baseline data used to fix the transport parameters are AMS-02 Li/C and B/C data (Aguilar et al. 2018) - denoted "base" in the following. The halo size $L$ is then constrained by combining the base with several ratios from
Table 2. Experiments with their data-taking periods and associated expected Solar modulation level.

\begin{tabular}{|c|c|c|}
\hline Experiment (period) & $\begin{array}{l}\phi_{\text {prior }} \\
{[\mathrm{MV}]}\end{array}$ & Reference \\
\hline \multicolumn{3}{|c|}{$\mathrm{Li} / \mathrm{C}$ and $\mathrm{B} / \mathrm{C}$ ("Base") } \\
\hline AMS-02 ('11/05-'16/05) & 676 & Aguilar et al. (2018) \\
\hline ACE-CRIS ('97/08-'98/04) & 528 & Lave et al. (2013) \\
\hline ACE-CRIS ('98/01-'99/01) & 582 & de Nolfo et al. (2006) \\
\hline ACE-CRIS ('01/05-'03/09) & 872 & Lave et al. (2013) \\
\hline ACE-CRIS ('09/03-'10/01) & 445 & Lave et al. (2013) \\
\hline IMP8 ('74/01-'78/10) & 540 & Garcia-Munoz et al. (1987) \\
\hline ISEE3-HKH ('78/08-'81/04) & 742 & Krombel \& Wiedenbeck (1988) \\
\hline Ulysses-HET ('90/10-'95/07) & 732 & Duvernois et al. (1996) \\
\hline Voyager1\&2 ('77/01-'98/12) & 450 & Lukasiak (1999) \\
\hline \multicolumn{3}{|c|}{$\mathrm{Be} / \mathrm{B}$ data } \\
\hline AMS-02 ('11/05-'16/05) & 676 & Aguilar et al. (2018) \\
\hline \multicolumn{3}{|c|}{${ }^{10} \mathrm{Be} / \mathrm{Be}$ data } \\
\hline IMP7\&8 ('72/09-'75/09) & 543 & Garcia-Munoz et al. (1977) \\
\hline IMP7\&8 ('74/01-'80/05) & 580 & Garcia-Munoz et al. (1981) \\
\hline ISEE3-HKH ('78/08-'79/08) & 653 & Wiedenbeck \& Greiner (1980) \\
\hline Ulysses-HET ('90/10-'97/12) & 661 & Connell (1998) \\
\hline Voyager1\&2 ('77/01-'98/12) & 450 & Lukasiak (1999) \\
\hline \multicolumn{3}{|c|}{${ }^{10} \mathrm{Be} /{ }^{9} \mathrm{Be}$ data } \\
\hline \multicolumn{3}{|c|}{ Most precise low-energy data ("ACE”) } \\
\hline ACE-CRIS ('97/08-'99/07) & 581 & Yanasak et al. (2001) \\
\hline ACE-SIS ('97/08-'99/07) & 581 & Yanasak et al. (2001) \\
\hline \multicolumn{3}{|c|}{ Remaining low-energy data ("LE w/o ACE") } \\
\hline IMP7\&8 ('74/01-'80/05) & 580 & Garcia-Munoz et al. (1981) \\
\hline ISEE3-HKH ('78/08-'79/08) & 653 & Wiedenbeck \& Greiner (1980) \\
\hline Ulysses-HET ('90/10-'97/12) & 661 & Connell (1998) \\
\hline Voyager1\&2 ('77/01-'98/12) & 450 & Lukasiak (1999) \\
\hline \multicolumn{3}{|c|}{ Intermediate energy } \\
\hline ISOMAX ('98/08) & & Hams et al. (2004) \\
\hline \multicolumn{3}{|c|}{ Preliminary data (Appendix B only) } \\
\hline PAMELA ('06/07-'14/09) & 500 & Bogomolov \& Vasilyev (2019) \\
\hline
\end{tabular}

Notes. In the list below, datasets from the same experiment and data taking periods share a common Solar modulation level in the analyses. PAMELA data are from a preliminary analysis. For this reason, they are never considered in our main results, and are only used for illustration in Appendix B.

different datasets. The largest dataset is that of AMS-02 Be/B, covering $\sim 3 \mathrm{GV}$ to $\sim 2 \mathrm{TV}$. We also have several low-energy ${ }^{10} \mathrm{Be} /{ }^{9} \mathrm{Be}$ or ${ }^{10} \mathrm{Be} / \mathrm{Be}$ datasets available, retrieved from $\mathrm{CRDB}^{2}$ (Maurin et al. 2014). Except for the ISOMAX data reaching $\sim 2 \mathrm{GeV} / \mathrm{n}$, most of them (ACE, IMP7\&8, ISEE3, Ulysses, and Voyager1\&2) are at a few hundreds of $\mathrm{MeV} / \mathrm{n}$.

The various datasets of interest are listed in Table 2, along with their estimated Solar modulation level and bibliographic reference. Several ratio and dataset combinations are considered, in order to assess and compare their respective impact on L. Combined to "base" (i.e. Li/C and B/C data only), which enables the determination of the transport parameters, at least one ${ }^{10} \mathrm{Be}$-related dataset is necessary to determine $L$. In particular, for fits involving ${ }^{10} \mathrm{Be} /{ }^{9} \mathrm{Be}$, we differentiate three groups (see Table 2): the most precise low-energy data only ("ACE"), low-energy data without ACE ("LE w/o ACE"), or intermediate energy data only ("ISOMAX"). For consistency for the Solar modulation nuisance parameters, in all fits involving low-energy isotopic ratios, we consider, if available, the associated similarly modulated $\mathrm{Li} / \mathrm{C}$ and $\mathrm{B} / \mathrm{C}$ low-energy data (in addition to AMS02 data).

\footnotetext{
2 https://lpsc.in2p3.fr/crdb
} 


\subsection{Expected constraints from $\mathrm{B} / \mathrm{C}, \mathrm{Be} / \mathrm{B}$, and ${ }^{10} \mathrm{Be} / \mathrm{Be}$}

Before moving to the fits and results, we wish to understand how strongly $L$ can be constrained by different data combinations of ${ }^{10} \mathrm{Be}$. Ratios directly involving ${ }^{10} \mathrm{Be}$ should be optimal, but owing to the experimental difficulty of achieving isotopic separation, high-precision elemental ratios like $\mathrm{Be} / \mathrm{B}$ can be competitive (Webber \& Soutoul 1998). In principle, B/C might also lead to some constraint, via the fraction of ${ }^{10} \mathrm{~B}$ generated by ${ }^{10} \mathrm{Be}$ decay. The most favourable option would include some information on the ${ }^{10} \mathrm{Be}$ content, high-precision data, and significant dynamic range. For instance, at $\sim 1 \mathrm{GeV} / \mathrm{n}$, the $\mathrm{B} / \mathrm{C}$ and $\mathrm{Be} / \mathrm{C}$ fraction from unstable isotopes are respectively $\sim 5 \%$ and $5-10 \%$, with data available from $\mathrm{GV}$ to $\mathrm{TV}$ at $3-5 \%$ precision (Aguilar et al. 2018); for ${ }^{10} \mathrm{Be}$, the fraction is $100 \%$ with data in the $\sim 50-200 \mathrm{MeV} / \mathrm{n}$ range at $\sim 10-15 \%$ precision (Yanasak et al. 2001).

We first pick a reference configuration and $L$ to which other calculations are compared. This configuration is taken from our companion paper, Weinrich et al. (2020), and is based on the simultaneous analysis of $\mathrm{AMS}-02 \mathrm{Li} / \mathrm{C}, \mathrm{Be} / \mathrm{C}$ and $\mathrm{B} / \mathrm{C}$ data at fixed $L=5 \mathrm{kpc}$. To see how sensitive to $L$ calculated ratios are, we vary $L$ ensuring that the level of production remains unchanged for secondary stable species-this constraint is satisfied with an appropriate rescaling of the transport parameters (see Appendix $\mathrm{A})^{3}$. From this set-up, we calculate the relative variation of $\mathrm{B} / \mathrm{C}, \mathrm{Be} / \mathrm{B},{ }^{10} \mathrm{Be} / \mathrm{Be}$, and ${ }^{10} \mathrm{Be} /{ }^{9} \mathrm{Be}$ with respect to the reference values at $L=5 \mathrm{pc}$.

The results are shown in Fig. 1, with the dependence upon growing $L$ illustrated via the growing thickness of dotted lines, while different uniformly coloured bands represent different theoretical uncertainties (described and discussed in Sect. 3.3). For $\mathrm{B} / \mathrm{C}$ (top panel), even for unrealistic variations of $L$, the maximal impact is a few percent only. On the other hand, varying $L$ from 2.5 to $12 \mathrm{kpc}$ leads to variations of up to $5 \%$ for $\mathrm{Be} / \mathrm{B}$ ( $\mathrm{sec}-$ ond panel) and up to $35 \%$ for ${ }^{10} \mathrm{Be} /{ }^{9} \mathrm{Be}$ and ${ }^{10} \mathrm{Be} / \mathrm{Be}$ (two bottom panels). Secondly, whereas these variations peak strongly around $\sim 30 \mathrm{GV}$ for $\mathrm{Be} / \mathrm{B}$, they are constant for all energy below a few $\mathrm{GeV} / \mathrm{n}$ for ${ }^{10} \mathrm{Be} /{ }^{9} \mathrm{Be}$ and ${ }^{10} \mathrm{Be} / \mathrm{Be}$. We overlay on the plots (hatched regions) the existing data coverage in terms of energy range and total uncertainties. From the comparison with the $L$-dependent curves (thin- to thick-dotted lines), we conclude that $\mathrm{B} / \mathrm{C}$ data alone can only provide very loose upper limits $\left(\lesssim 20-30 \mathrm{kpc}\right.$ ), while $\mathrm{Be} / \mathrm{B},{ }^{10} \mathrm{Be} /{ }^{9} \mathrm{Be}$, and ${ }^{10} \mathrm{Be} / \mathrm{Be}$ are all expected to constrain $L$ to better than a factor 2 .

\subsection{Directions for future experimental efforts}

By pursuing further the reasoning, we can also forecast where future measurements could improve the constraint on $L$. From Fig. 1, energy ranges where $L$ is the most impacting are between 10 and $100 \mathrm{GV}$ for $\mathrm{Be} / \mathrm{B}$ data, and below a few $\mathrm{GeV} / \mathrm{n}$ for ${ }^{10} \mathrm{Be} /{ }^{9} \mathrm{Be}$. However, to fairly assess the sensitivity of each ratio, model uncertainties should be taken into account-broadly speaking, model uncertainties originate from transport, cross-section, and solar modulation.

Technically we proceed as follows: starting from the best fit and covariance matrix of the relevant parameters (standard outputs of fits with USINE v3.5), we draw realisations of the param-

\footnotetext{
3 There is a subtlety for $\mathrm{B} / \mathrm{C}$, as it contains ${ }^{10} \mathrm{~B}$ having decayed from ${ }^{10} \mathrm{Be}$. Depending how scaling relations are derived, they absorb or not the $L$ dependence of this decayed fraction. We use the theory-based scaling relations to study the sensitivity of $\mathrm{B} / \mathrm{C}$ to $L$, but the fit-driven ones for the remaining ratios (see Appendix A).
}
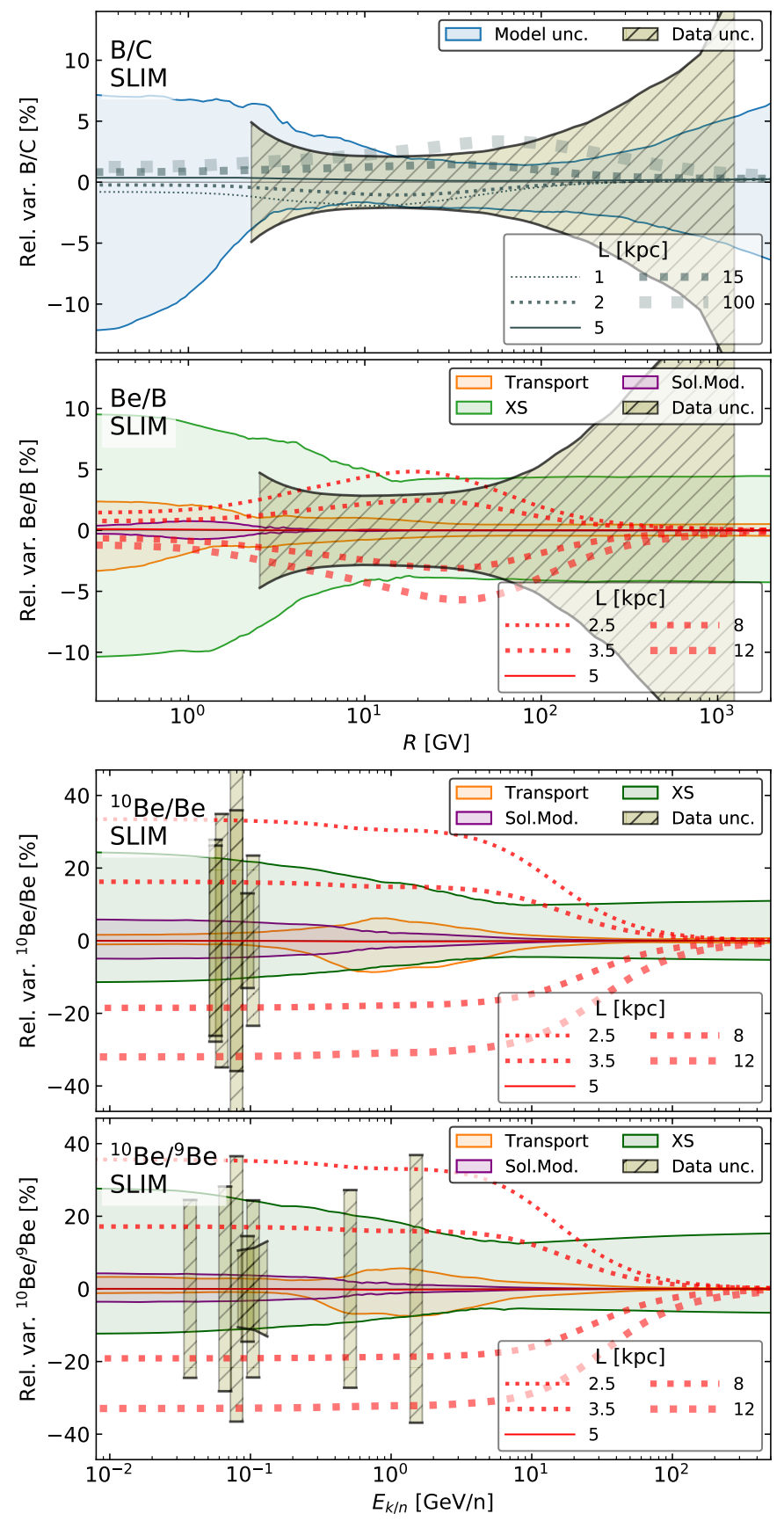

Fig. 1. Relative variation of $\mathrm{B} / \mathrm{C}, \mathrm{Be} / \mathrm{B},{ }^{10} \mathrm{Be} / \mathrm{Be}$, and ${ }^{10} \mathrm{Be} /{ }^{9} \mathrm{Be}$ ratios (from top to bottom) as a function of rigidity (upper panels) or kinetic energy per nucleon (lower panels). All the panels compare three different effects: (i) the thin- to thick-dashed lines correspond, at constant grammage, to the impact of $L$ on the calculated ratios (the reference is $L=5 \mathrm{kpc}$ ); (ii) hatched boxes show the data relative uncertainties over the data energy coverage (AMS-02 data in the two top panels) or for central energy points only (ACE, IMP7 \&8, ISEE3, ISOMAX, Ulysses, and Voyager1\&2 data in the two bottom panels) - see Sect. 3.1 and Table 2 for data references; (iii) the remaining envelopes show $68 \%$ CLs on model calculations, including transport, cross sections, and modulation uncertainties separately or combined ("total").

eters of interest, from which new values for the ratios are calculated; we then extract contours and confidence levels on these ratios. Repeating the drawing procedure considering the full covariance matrix of parameters or only block elements of this 
matrix, we can propagate the model uncertainties all together (e.g. B/C in the top panel of Fig. 1) or separately (all remaining panels). This naturally accounts for the full or partial correlations between the parameters.

We show in the panels of Fig. 1 various model uncertainties ( $1 \sigma$ contours) obtained from sampling $10^{4}$ realisations of the model parameters for SLIM ${ }^{4}$. Overall, solar modulation uncertainties ( $\lesssim 5 \%$, purple contours) are sub-dominant in the model error budget; they are also sub-dominant with respect to data uncertainties (hatched boxes). For isotopic ratios, cross-section uncertainties dominate $(\sim 10-20 \%$, green contours $)$, followed by transport uncertainties ( $\lesssim 10 \%$, orange contours). Let us detail separately the conclusions that can be drawn for $\mathrm{B} / \mathrm{C}, \mathrm{Be} / \mathrm{B}$, ${ }^{10} \mathrm{Be} / \mathrm{Be}$, and ${ }^{10} \mathrm{Be} /{ }^{9} \mathrm{Be}$ - for completeness, we also show in Appendix $\mathrm{C}$ the ${ }^{7} \mathrm{Be} /\left({ }^{9} \mathrm{Be}+{ }^{10} \mathrm{Be}\right)$ ratio, more easily measured but unfortunately not constraining for $L$.

Firstly, the status of the $\mathrm{B} / \mathrm{C}$ ratio (top panel) is different from the other ones, because transport is calibrated on it. For this reason, the associated uncertainties are typically at the level of the data uncertainties. Given the poor sensitivity of $\mathrm{B} / \mathrm{C}$ to $L$, at least a factor of ten improvement on data errors would be necessary to bring any improvement on $L$.

Secondly, for the $\mathrm{Be} / \mathrm{B}$ ratio (second panel), the optimal region to constrain $L$ is from a few $\mathrm{GV}$ to a few tens of $\mathrm{GV}$, exactly in the region where AMS-02 data have the smallest errors $(\sim 3 \%)$. In this region, cross-section uncertainties (green contours) are at the 5\% level, meaning that better nuclear crosssection data could already slightly shrink the allowed $L$ values by $\sim 30 \%$. Better Be/B data could strengthen the $L$ constraint, but only with cross-sections uncertainties brought to par. However, improving CR data systematics below the percent level is very challenging.

Thirdly, for the isotopic ${ }^{10} \mathrm{Be} / \mathrm{Be}$ and ${ }^{10} \mathrm{Be} /{ }^{9} \mathrm{Be}$ ratios (bottom two panels), we have to slightly change our estimation of crosssection uncertainties. Contrarily to elemental ratios, in which only the overall element production matters (sum of ${ }^{7} \mathrm{Be},{ }^{9} \mathrm{Be}$, and ${ }^{10} \mathrm{Be}$ ), isotopic ratios directly depend on the associated isotopic production cross sections. To be conservative - though probably too pessimistic -, we draw each isotopic production cross sections (independently) within their expected uncertainty range $^{5}$. As for $\mathrm{Be} / \mathrm{B}$, the cross-section uncertainties are the dominant modelling uncertainties. At variance with $\mathrm{Be} / \mathrm{B}$, any region below $10 \mathrm{GeV} / \mathrm{n}$ is equally suited to constrain $L$. In the highenergy end of this interval, current data uncertainties are larger than the model uncertainties, so that there is a small window for improvements on data to improve the constraints on $L$. Also, CR data on a large energy range should provide a better lever arm to handle cross-section uncertainties.

We can now conclude on the best way to improve the constraints on $L$ in the future. On the short term, forthcoming ${ }^{10} \mathrm{Be} /{ }^{9} \mathrm{Be}$ AMS-02 and PAMELA data up to $10 \mathrm{GeV} / \mathrm{n}$ are the best candidates to improve the constraints on $L$. On a longer term, the easiest way to improving isotopic CR data would be to focus on $\mathrm{GeV} / \mathrm{n}$ energies for this same ratio. Improving the precision of $\mathrm{Be} / \mathrm{B}$ data would be only significant at the sub-percent level, but this is likely to remain difficult to achieve, even for future

\footnotetext{
4 Configurations having more free parameters (QUAINT and BIG) would provide larger uncertainties; see Fig. 4 in Weinrich et al. (2020).

5 We follow the NSS prescription (Derome et al. 2019; Weinrich et al. 2020), where we vary the cross-section normalisation and low-energy behaviour (power-law slope) of the dominant production channel for each $\mathrm{Be}$ isotope. We use a dispersion $\sigma_{\text {slope }}=0.15$ for these reactions, and $\sigma_{\text {Norm }}=0.25,0.20$, and 0.15 for ${ }^{12} \mathrm{C}+\mathrm{H} \rightarrow{ }^{10} \mathrm{Be},{ }^{12} \mathrm{C}+\mathrm{H} \rightarrow{ }^{9} \mathrm{Be}$, and ${ }^{16} \mathrm{O}+\mathrm{H} \rightarrow{ }^{7} \mathrm{Be}$ respectively.
}

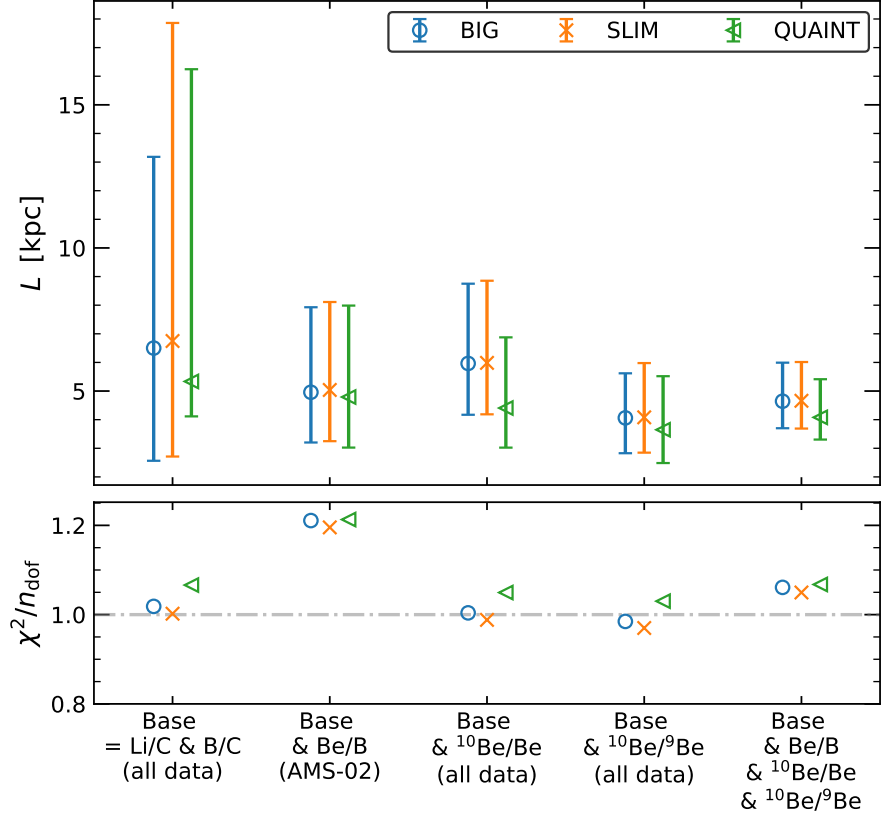

Fig. 2. Best-fit halo size and asymmetric uncertainties (from MINOS) for the configurations BIG, SLIM, and QUAINT. From left to right, several data combinations are used. The first column ("Base") involves AMS-02 and low-energy $\mathrm{B} / \mathrm{C}$ and $\mathrm{Li} / \mathrm{C}$ data. The second column is the constraint set from AMS- 02 data only $(\mathrm{Li} / \mathrm{C}, \mathrm{Be} / \mathrm{B}$, and $\mathrm{B} / \mathrm{C})$. The remaining columns combine "Base" data (from first column) to ${ }^{10} \mathrm{Be} / \mathrm{Be}$ low-energy data (third column), to ${ }^{10} \mathrm{Be} /{ }^{9} \mathrm{Be}$ low- and intermediateenergy (next-to-last column), or combine all the previous cases (last column).

CR projects like HERD (Cattaneo \& HERD Collaboration 2019), ALADInO (Adriani et al. 2019), or AMS-100 (Schael et al. 2019). In any case, be it for $\mathrm{Be} / \mathrm{B}$ or isotopic ratios, the common limiting factor to all improvements are cross-section uncertainties.

\subsection{Actual constraints on $L$}

In this section, we present the constraints on $L$ from various data combinations. The fitting procedure and free (and nuisance) parameters are as discussed in Sect. 2 - see also our companion paper for more details (Weinrich et al. 2020).

\subsubsection{Results}

Figure 2 shows the constraints (at $1 \sigma$ ) brought by the ratios discussed in the previous section $\left(\mathrm{B} / \mathrm{C}, \mathrm{Be} / \mathrm{B},{ }^{10} \mathrm{Be} / \mathrm{Be}\right.$, and ${ }^{10} \mathrm{Be} /{ }^{9} \mathrm{Be}$ ) for the three configurations BIG (blue circle), SLIM (orange crosses), or QUAINT (green triangles). We first stress that the various transport configurations all give similar constraints on $L$ - we do not show results for the transport parameters as they are available and were abundantly discussed in Weinrich et al. (2020). The behaviour for the different ratios (columns) is in qualitative agreement with the expectations discussed in Sect. 3.2. In the first column, the $\mathrm{B} / \mathrm{C}$ ratio mostly gives an upper limit $L \lesssim 15 \mathrm{kpc}$, whereas in the second and third columns, Be/B AMS-02 and ${ }^{10} \mathrm{Be} / \mathrm{Be}$ low-energy data give similar constraints $L \approx 5 \pm 3 \mathrm{kpc}$. The fourth column is based on the results from the same experiments as in the third column, but fitting ${ }^{10} \mathrm{Be} / \mathrm{Be}$ instead of ${ }^{10} \mathrm{Be} /{ }^{9} \mathrm{Be}$ data, and with the additional use of intermediate-energy ISOMAX data (see Table 2). This gives a slightly lower best-fit value and uncertainties compared 
Table 3. Halo size fit results for the combined analysis of $\mathrm{Li} / \mathrm{C}$ and $\mathrm{B} / \mathrm{C}$ (denoted "Base", see also Fig. 2) with an "unstable-to-stable" secondary ratio $r$.

\begin{tabular}{lccc}
\hline \hline & BIG & SLIM & QUAINT \\
\hline \multicolumn{4}{c}{ Base \& Be/B } \\
(AMS-02) \\
$L[\mathrm{kpc}]$ & $4.96_{-1.76}^{+2.97}$ & $5.04_{-1.79}^{+3.07}$ & $4.79_{-1.77}^{+3.19}$ \\
$\chi^{2} / n_{\text {d.o.f. }}$ & $233.7 / 193$ & $233.1 / 195$ & $235.3 / 194$ \\
$\chi_{\text {nui }}^{2} / n_{\text {nui }}$ & $17.4 / 20$ & $17.4 / 20$ & $15.8 / 20$ \\
\hline \multicolumn{4}{c}{ Base $\& \mathrm{Be} / \mathrm{B} \mathrm{\&}{ }^{10} \mathrm{Be} / \mathrm{Be} \&{ }^{10} \mathrm{Be} /{ }^{9} \mathrm{Be}$} \\
$($ all data) \\
$L[\mathrm{kpc}]$ & $4.64_{-0.94}^{+1.35}$ & $4.66_{-0.97}^{+1.35}$ & $4.08_{-0.78}^{+1.33}$ \\
$\chi^{2} / n_{\text {d.o.f. }}$ & $266.3 / 251$ & $265.6 / 253$ & $269.0 / 252$ \\
$\chi_{\text {nui }}^{2} / n_{\text {nui }}$ & $25.6 / 35$ & $25.4 / 35$ & $25.6 / 35$ \\
\hline
\end{tabular}

Notes. The top rows show the constraint from AMS-02 data $(r=\mathrm{Be} / \mathrm{B})$, while the bottom rows show the combined constraint from all available datasets $\left(r=\mathrm{Be} / \mathrm{B}+{ }^{10} \mathrm{Be} / \mathrm{Be}+{ }^{10} \mathrm{Be} /{ }^{9} \mathrm{Be}\right)$.

to ${ }^{10} \mathrm{Be} / \mathrm{Be}$ data, but this apparent improvement is related to a tension between ISOMAX data and all the others. The reduced uncertainties result from an attempt to accommodate all the data at once - Appendix B details results on the broken-down constraints from various low-energy datasets. The tension with ISOMAX data also reflects in the global fit (last column), which is pushed towards slightly larger $L$ values, also preferred by AMS$02 \mathrm{Be} / \mathrm{B}$ data (second column).

We gather in Table 3 the best-fit values and $1 \sigma$ uncertainties on $L$ for the AMS-only analysis (with $\mathrm{Be} / \mathrm{B}$, top) and the combined analysis (with $\mathrm{Be} / \mathrm{B}$ and all isotopic ratios, bottom). In terms of the $\chi_{\min }^{2} /$ d.o.f. values, a fair but not perfect agreement is obtained when using AMS-02 only data $\left(\chi_{\min }^{2} /\right.$ d.o.f. $\left.\sim 1.2\right)$. An excellent fit is obtained for the isotopic data with $\chi_{\min }^{2} /$ dof $~$ 1.0, and also when combining elemental and isotopic data with $\chi_{\min }^{2}$ /d.o.f. $\sim 1.06$ (last column in Fig. 2 or bottom of Table 2); for the latter, low-energy $\mathrm{Li} / \mathrm{C}, \mathrm{B} / \mathrm{C}$, and also ${ }^{10} \mathrm{Be}$-related ratios are in good agreement with the constraints set by AMS-02 data only and thus merely increases $n_{\text {data }}$ without increasing $\chi_{\min }^{2}$. The last row in Table 3 shows the value of

$\chi_{\text {nui }}^{2} / n_{\text {nui }} \equiv\left(\sum_{s=0}^{n_{s}} \mathcal{N}_{\text {Sol.Mod. }}^{s}+\sum_{x=0}^{n_{x}} \mathcal{N}_{\mathrm{XS}}^{x}\right) /\left(n_{s}+n_{x}\right)$,

with $\mathcal{N}_{\text {Sol.Mod. }}^{s}$ and $\mathcal{N}_{\mathrm{XS}}^{x}$ the $n_{s}$ and $n_{x}$ nuisance parameters for solar modulation and cross sections respectively $\left(n_{\text {nui }}=n_{s}+n_{x}\right)$. As discussed in Weinrich et al. (2020), this quantity gives a direct check that nuisance parameters behave properly. On average, nuisance parameters post-fit values should never be more than $1 \sigma$ away from their prior, that is, $\chi_{\text {nui }}^{2} / n_{\text {nui }} \lesssim 1$, and this is verified for all our fits.

For illustration purposes, we finally show in Figs. 3 and 4 the model calculation and the data for $\mathrm{Be} / \mathrm{B}$ and isotopic ratios. The parameters are taken from the best-fit to all combined $\mathrm{Be} / \mathrm{B}$, ${ }^{10} \mathrm{Be} / \mathrm{Be}$, and ${ }^{10} \mathrm{Be} /{ }^{9} \mathrm{Be}$ data (last column in Fig. 2). In both plots, the top panels show the model calculations for the three transport configurations (BIG, SLIM, and QUAINT) along with the data. For SLIM, we also superimpose the $1 \sigma$ model total uncertainties (contours) as calculated in Sect. 3.3. The second panels illustrate the goodness-of-fit to the data via the residuals between the data and the model. For the Be/B case with AMS-02 data (Fig. 3), a third panel shows the "rotated" score $\tilde{z}$, as defined in Boudaud
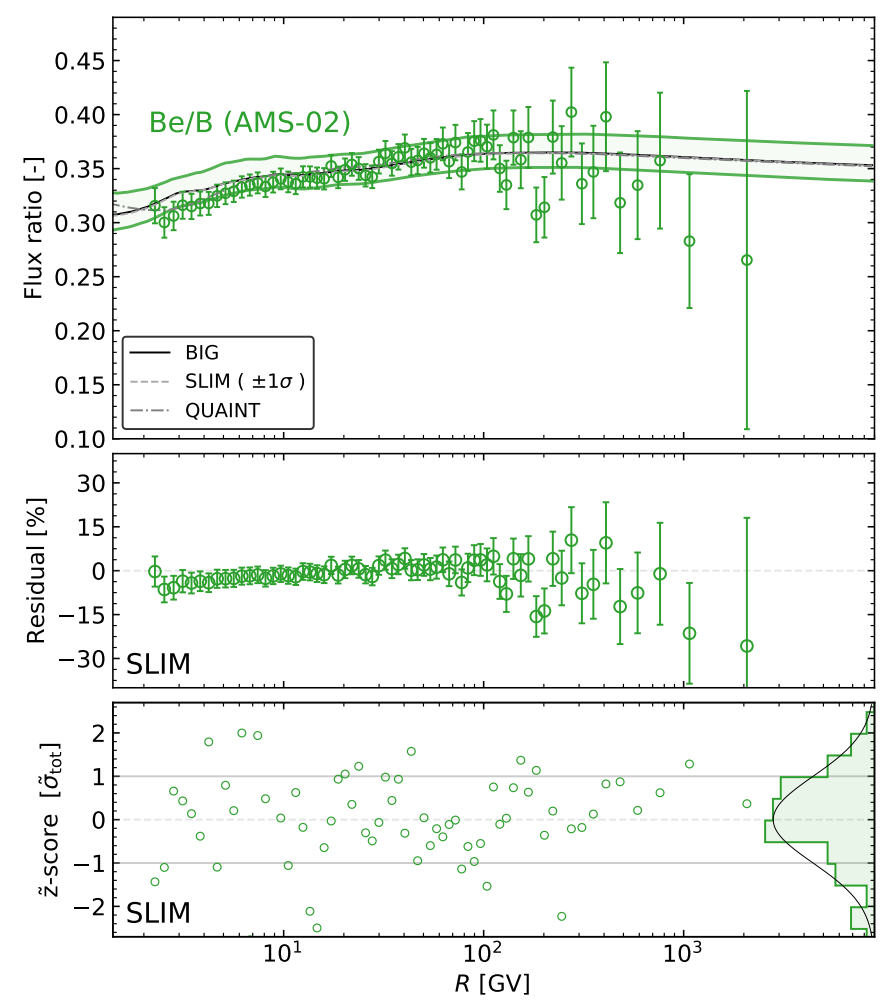

Fig. 3. Model prediction (top), residuals (centre), and ż-score (bottom) for $\mathrm{Be} / \mathrm{B}$ based on the best-fit parameters to $\mathrm{B} / \mathrm{C}, \mathrm{Li} / \mathrm{C},{ }^{10} \mathrm{Be} /{ }^{9} \mathrm{Be}$, ${ }^{10} \mathrm{Be} / \mathrm{Be}$ and $\mathrm{Be} / \mathrm{B}$ data. In the top panel, the contours show the $1 \sigma$ total model uncertainties for BIG. In the bottom panel, the right-hand side shows the distribution of $\tilde{z}$ values against a Gaussian with unit width (solid lines).

et al. (2020) or Weinrich et al. (2020): It suffices to say that this score represents an unbiased visual representation of the distance between the model and the data, accounting for existing rigidity correlations in the systematics of AMS-02 data; also, the chisquare is the sum of the squares of these rotated residuals, that is, $\chi_{\mathrm{Be} / \mathrm{B}}^{2}=\sum_{i} \tilde{z}_{i}^{2}$. The right-hand side of the bottom panel is another illustration of the goodness of fit of the model, for the distribution of $\tilde{z}$-values is expected to follow a Gaussian distribution of width one.

\subsubsection{Discussion}

It is interesting to compare our results to those of previous analyses that considered either ACE-CRIS ${ }^{10} \mathrm{Be} /{ }^{9} \mathrm{Be}$ ratio or $\mathrm{Be} / \mathrm{B}$ data. Based on the analysis of ${ }^{10} \mathrm{Be} /{ }^{9} \mathrm{Be}$ and other radioactive isotopes, and using a diffusion model with $\delta \approx 0.3$, the GALPROP team found $L \in$ [1.5-6] kpc (Moskalenko et al. 2001), and later on, using an evolved Bayesian analysis, found $L=5.4 \pm 1.4 \mathrm{kpc}$ (Trotta et al. 2011). Actually, the halo size strongly correlates with the diffusion slope $\delta$ (Donato et al. 2002; Putze et al. 2010). Using 1D or 2D semi-analytical models, our team found $L \approx 5 \mathrm{kpc}$ (Donato et al. 2002) for $\delta \sim 0.5$, and later on, also in an evolved Bayesian context, found $L \approx 4 \pm 1 \mathrm{kpc}$ in a pure diffusion/reacceleration model (Putze et al. 2010). All these values are consistent with the constraints derived here using ACE-CRIS ${ }^{10} \mathrm{Be} /{ }^{9} \mathrm{Be}$ data only (see Fig. 2 ), that is $L \in[3-8] \mathrm{kpc}$. Our uncertainties are larger than in previous studies, because we include here production cross-section uncertainties.

Comparatively, less studies focused on elemental ratios. Using HEAO-3 Be/B data (and other ratios) in a semi-analytical 


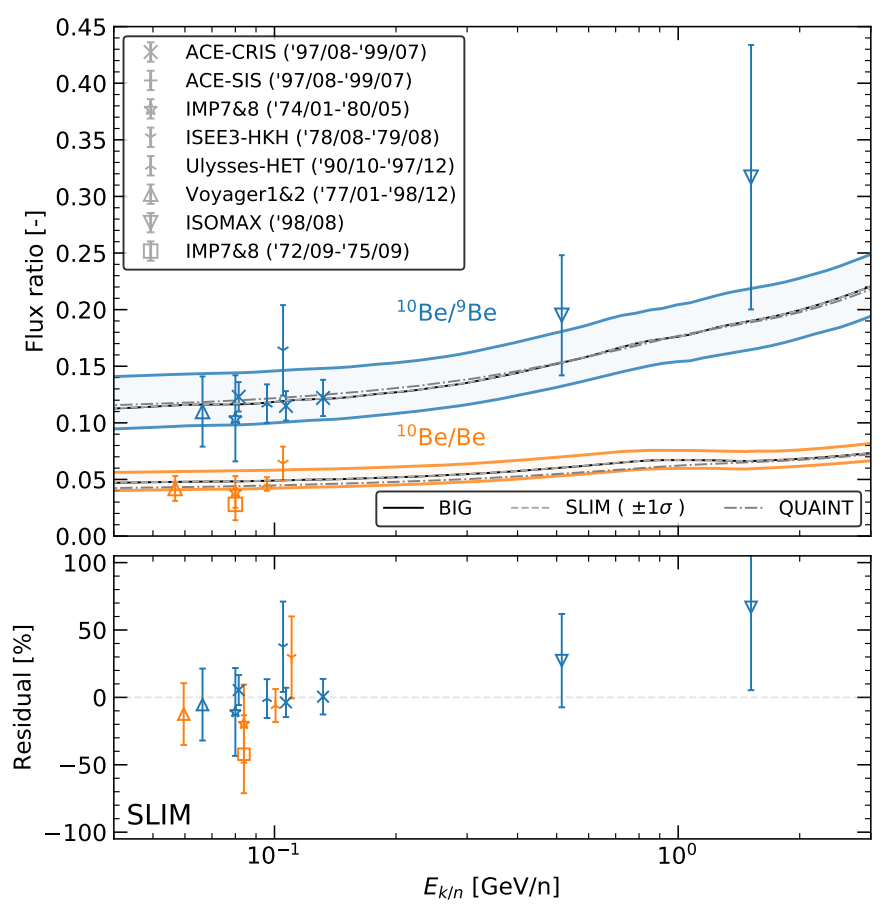

Fig. 4. Model prediction (top) and residuals (bottom) for ${ }^{10} \mathrm{Be} /{ }^{9} \mathrm{Be}$ (blue curves and symbols) and ${ }^{10} \mathrm{Be} / \mathrm{Be}$ (orange curves and symbols). In the top panel, the $1 \sigma$ contour corresponds to the total model uncertainties for SLIM (as calculated in Sect. 3.3). The model calculations are based on the parameters from the combined fit of all $\mathrm{Li} / \mathrm{C}, \mathrm{Be} / \mathrm{C}, \mathrm{Be} / \mathrm{B}$, ${ }^{10} \mathrm{Be} /{ }^{9} \mathrm{Be}$, and ${ }^{10} \mathrm{Be} / \mathrm{Be}$ data presented in Table 2 (PAMELA preliminary data excepted).

diffusion model with $\delta=0.6$, a rough range of $L \in[2-4] \mathrm{kpc}$ was found in Webber \& Soutoul (1998). A much larger range was found in Putze et al. (2010), with $L$ a few kpc only allowed for $\delta \lesssim 0.3$, growing to larger than $10 \mathrm{kpc}$ for $\delta \approx 0.5$. This tension with ${ }^{10} \mathrm{Be} /{ }^{9} \mathrm{Be}$ data was attributed to cross-section uncertainties - their importance in limiting the prediction power of $\mathrm{Be} / \mathrm{B}$ is detailed in Tomassetti (2015a). Using updated cross sections and propagating their uncertainties, we find here $L=$ $5_{-2}^{+3} \mathrm{kpc}$ based on AMS-02 Be/B data, which is now compatible with values derived from ACE-CRIS ${ }^{10} \mathrm{Be} /{ }^{9} \mathrm{Be}$ data. Similar values were found by the GALPROP team, using both ACE-CRIS and AMS-02 Be fluxes (without ${ }^{10} \mathrm{Be} /{ }^{9} \mathrm{Be}$ ), with $L=4 \pm 0.6 \mathrm{kpc}$ (Boschini et al. 2020). This stronger constraint is somehow in line with the fact that combining ACE-CRIS and AMS-02 data lead to tighter constraints (see Table 3), but as these authors do not use ${ }^{10} \mathrm{Be} /{ }^{9} \mathrm{Be}$, it is most probably attributed to a larger extent to the fact that they do not propagate cross-section uncertainties. In any case, the value of $L$ with a small error inferred in Boschini et al. (2020) is also in mild tension with the recent analysis of AMS-02 Be data in Evoli et al. (2020), where a best-fit value of $L=7 \mathrm{kpc}$ but a lower limit $L \gtrsim 5 \mathrm{kpc}$ is found in a semi-analytical model similar to the one used here. Their central value, using total or statistical uncertainties only, is always enclosed within our $1 \sigma$ confidence interval: firstly, the difference with our best-fit value is possibly due to their cross-section parametrisation (Evoli et al. 2019), as illustrated in Fig. 2 of Evoli et al. (2020); secondly, our broader confidence interval is certainly related to our treatment (full propagation) of crosssection uncertainties.

In conclusion, most past and present analyses of ${ }^{10} \mathrm{Be} /{ }^{9} \mathrm{Be}$ and $\mathrm{Be} / \mathrm{B}$ show a preference for $L \approx 4-5 \mathrm{kpc}$, with a currently estimated $1 \sigma$ uncertainty of about $50 \%$. Several analyses have combined or compared these results to the constraints brought by other ratios of radioactive secondary species (Webber \& Soutoul 1998; Moskalenko et al. 2001; Donato et al. 2002; Putze et al. 2010): similar halo sizes were observed, though with a large scatter. Forthcoming AMS-02 data on other elemental ratios (e.g. $\mathrm{Al} / \mathrm{Mg}, \mathrm{Cl} / \mathrm{Ar} . .$. ) will allow one to repeat these analyses. Another effect could impact these conclusions. In Donato et al. (2002) and Putze et al. (2010), the impact of a local subdensity, exponentially attenuating the flux of radioactive species, was inspected: slightly larger or much larger uncertainties were observed depending on the transport configuration used (Putze et al. 2010). However, this was calculated assuming a similar diffusion in the local bubble and in the rest of the disc and halo, which may be questionable. Recent $\gamma$-ray observations (Abeysekara et al. 2017; Di Mauro et al. 2019) - interpreted as pockets of slow diffusion around pulsars (e.g. Profumo et al. 2018) -, and the indication that the local ISM properties are affected by several SN explosions a few Myr ago (Fields et al. 2019) provide enough motivation to revisit this issue in a future study.

\section{Constraints on $L$ from other probes}

In this section, we discuss several independent constraints on $L$. We first review a series of constraints derived from multiwavelength observations of the Milky Way and other spiral galaxies, and then determine explicitly direct constraints induced by low-energy secondary CR positrons.

\subsection{Direct and indirect constraints from radio and gamma's}

Radio emission in the $\mathrm{MHz}$ to $\mathrm{GHz}$ band from our Galaxy has been used to constrain the magnetised halo thickness L (Bringmann et al. 2012; Orlando \& Strong 2013; Di Bernardo et al. 2013, and Biswas \& Gupta 2018). This band is dominated by the synchroton emission of the leptonic component of $\mathrm{CR}$ in the Galactic magnetic field. The Galactic latitude profile of the radio maps are sensitive to the vertical gradient of CR sourcing the emission, $\propto 1 / L$. Although relying on some assumptions (mostly on the magnetic field) these arguments tend to agree in excluding low values of $L$, typically obtaining $L \gtrsim 2 \mathrm{kpc}$, with variations within a factor 2 depending on the analysis. They are somewhat less sensitive to large values of $L$, although upper limits in the range $L \lesssim 10-15 \mathrm{kpc}$ have been derived. Further arguments based on radio observations such as rotation measurements of pulsars also yield results broadly consistent with these constraints (Di Bernardo et al. 2013).

A complementary indirect indication can be derived by looking at the radio emissions of other spiral galaxies seen almost edge-on, which present an average scale-height of their synchrotron emission of about $1.8 \pm 0.2 \mathrm{kpc}$ (Krause 2014). This translates, under the hypothesis of energy equipartition between magnetic field and cosmic ray energy density, in the typical constraint on the magnetised halo size $L \gtrsim 6.2-7.8 \mathrm{kpc}$ (Beck 2015). A recent analysis of an in-depth view of a spiral galaxy very similar to the Milky Way, NGC 891, reaches similar conclusions as for the vertical extension of the magnetic halo (Schmidt et al. 2019).

In principle, independent constraints can be derived from diffuse Galactic $\gamma$-ray data (Stecker \& Jones 1977). The advantage is that the bulk of the data in the Fermi-LAT energy range $(\mathrm{GeV})$ comes from $\pi^{0}$ decays of hadronic origin. Hence, the $\gamma$-ray flux mostly probes the convolution of the hadronic CR 
flux with the gas density. For instance, let us consider a simple model where CR transport is purely diffusive within a slab (1D-model). Assuming the gas is exponentially distributed with typical thickness $h$, the photon flux originating from the Galactic zenith scales as:

$$
\begin{aligned}
\Phi_{\gamma} & \propto \int_{0}^{\infty} \mathrm{d} z \Phi_{p}(z) n_{\mathrm{gas}}(z) \\
& \propto \Phi_{p}(z=0) \int_{0}^{L} \mathrm{~d} z\left(1-\frac{z}{L}\right) \exp \left(-\frac{z}{h}\right) \\
& \propto 1-\frac{h}{L}+O\left(\frac{h^{2}}{L^{2}}\right) .
\end{aligned}
$$

Since we expect $h / L \sim 1 \%-10 \%$, the factors entering the actual $\gamma$-ray flux normalisation should be known to an unrealistically high precision for this method to provide meaningful constraints on $L$. However, additional inverse Compton contributions from leptonic CR component are also sensitive to $L$, and cannot be neglected. A global analysis of the diffuse $\gamma$-ray emission measured by the Fermi-LAT satellite actually shows a systematic improvement of the statistical likelihood as $L$ increases up to $\sim 10 \mathrm{kpc}$ (Ackermann et al. 2012), an effect particularly important when fitting the emission at large longitudes. Conversely, the study of high- and intermediate-velocity clouds at few kpcs away from the Galactic plane leads to the constraint $L<6 \mathrm{kpc}$ (Tibaldo et al. 2015). Although this bound can be subject to variations (e.g. from the presence of unaccounted ionised gas in these clouds), the different trends between this constraint and the one obtained from radio observations of distant galaxies might be explained by the radio emission of leptons leaking out beyond the confinement volume. Also, the too shallow $\gamma$-ray gradient suggested by Fermi-LAT data admits alternative explanations, for instance in terms of a physically motivated correlation of the diffusion properties with galactocentric distance (Evoli et al. 2012).

\subsection{Constraints from $e^{+}$}

Secondary positrons, usually believed to dominate the local CR positron flux at low energy, can also be used to derive lower limits on the halo size (Lavalle et al. 2014; Boudaud et al. 2017; Reinert \& Winkler 2018). Their predicted abundance depends much less on the configuration of the Galactic magnetic field than the radio limits, and their theoretical uncertainties are better under control (see below). Secondary positrons may therefore significantly constrain propagation models, while still on the conservative side since their local flux is known to be dominated by primaries above a few $\mathrm{GeV}$ (e.g. Aharonian et al. 1995; Adriani et al. 2009; Hooper et al. 2009; Delahaye et al. 2010).

\subsubsection{Positron flux scaling with $K_{0}$ and $L$ and calculation}

The steady-state local positron density is expected to scale like the production rate times the minimal propagation timescale involved. An additional dilution factor comes from the fact that the production volume $V_{\mathrm{p}}$ is, in most cases, smaller than the diffusion volume $V_{\lambda}$ (Bulanov et al. 1976). Sticking to a one-dimensional picture, we have $V_{\mathrm{p}} / V_{\lambda} \approx h / \lambda(E)$. In the latter expression, $h$ is the half-height of the thin disc (where the ISM gas is confined), and $\lambda(E)=\sqrt{2 K_{0} \tilde{\tau}(E)}$ is the positron propagation length scale, featuring the pseudo-energy loss timescale $\tilde{\tau}$.
The interesting regime to constrain $L$ is, similarly to radioactive species, when $h<\lambda<L$, that is, when the vertical boundary does not affect the positron density and $\tilde{\tau}$ does not depend appreciably on $K_{0}$. This typically happens at energies $\lesssim 10 \mathrm{GeV}$, for which $\lambda \propto \sqrt{K_{0}}$, and the $\mathrm{e}^{+}$flux then scales as $1 / \sqrt{K_{0}}$. Therefore, since energy loss parameters are fixed independently from the propagation model, the positron flux is a direct probe of the diffusion coefficient normalisation $K_{0}$. Since the B/C ratio provides constraints on the ratio $K_{0} / L$, positron measurements enable an indirect probe of $L$ : The lower $L$ in a B/C-compatible model, the larger the secondary positron flux.

In practice, we calculate the positron flux according to the pinching method introduced in Boudaud et al. (2017) - see also Delahaye et al. (2009) for earlier attempts. For production, we consider incident and target species up to He only, and we take the cross-section parametrisation of Kamae et al. (2006), which accounts for the low-energy hadronic resonances. Positron fluxes are then compared to AMS-02 data (Aguilar et al. 2019a) assuming $\phi_{\mathrm{FF}}=650 \mathrm{MV}$, as estimated for the corresponding data-taking period May 2011-November 2017 (see below for a discussion on Solar modulation level and its uncertainties). There are various sources of uncertainties in the calculation, and we try to list and quantify them below.

\subsubsection{Error budget}

We consider the uncertainties on the interstellar (IS) flux, and then comment on how the conclusions change for Top-ofAtmosphere (TOA) fluxes. We mostly focus on results at $1 \mathrm{GeV}$, because this is the typical energy where our analysis can draw constraints (see next subsection).

Firstly, we consider model uncertainties. Indeed, the method of calculation itself has some limitations. In the low- and highenergy regimes, propagation is dominated by energy losses in the disc and in the halo respectively. The pinching method allows to calculate intermediate energies by pinching the halo losses in the disc (Boudaud et al. 2017), ensuring that both the limiting cases are recovered. Further comparisons against full numerical solutions should be carried out to definitively assess the accuracy of the method in the transition zone. Nevertheless, we have checked that the method is robust in the energy range used to define our limits below.

In a broader context, one could question the reliability of 1D models for consistency checks between nuclei and leptons, in the context of spatially-dependent distributions of sources and gas. Given the timescales of various transport parameters and energy losses, both these species originate from a few kpc away at $\mathrm{GeV}$ energies (Taillet \& Maurin 2003; Maurin \& Taillet 2003; Jóhannesson et al. 2016). It means that their production and losses are sensitive to kpc-averages over the gas density properties - of course, this is no longer the case for very high energy leptons. So as long as we focus on the multi-GeV energy range, we do not expect strong differences due to gas inhomogeneities between nuclei and positrons, but as would be expected from more refined models, 1D model calculations are sensitive to the absolute value of the averaged gas density. This is at variance with the case of secondary radioactive species, discussed in Sect. 3.4, which could be very sensitive to the local ISM. However, similarly to radioactive nuclei, inhomogeneous spatial diffusion zones around CR sources (Abeysekara et al. 2017; Di Mauro et al. 2019), could also affect primary and secondary lepton spectra in different and very non trivial ways. These complications go beyond the scope of this analysis. 
Secondly, we consider uncertainties from the choice of CR projectiles, targets, and cross sections. Any uncertainty on the CR fluxes and production cross sections directly impact the number of secondary positrons. For CR fluxes, we take demodulated proton and helium CR fluxes measured by AMS02 (Aguilar et al. 2015, 2017), and these data typically have uncertainties in the 3-10\% range. Also, not accounting for the production from heavier CRs and species heavier than $\mathrm{He}$ in the ISM underestimates the secondary positron flux. Following the detailed analysis of Boudaud et al. (2020) carried out for antiprotons, we can estimate these effects to be $\sim 10 \%$ and $\sim 3 \%$ respectively.

Concerning production cross-section uncertainties, we recall that we use Kamae et al. (2006) parametrisation. More recent values exist (Kachelrieß et al. 2019) - they are calibrated on more recent collider data and include incident and target species up to $\mathrm{Fe}-$, but they are only valid for incident nucleus energy greater than $4 \mathrm{GeV}$. Our analysis is mostly sensitive to the low-energy part, so the latter model is only used to get a rough estimate of the theoretical uncertainties in the production cross sections. The secondary positron flux is $10-20 \%$ larger with Kachelrieß et al. (2019) than with Kamae et al. (2006) values.

Thirdly, we consider uncertainties from energy loss modelling. Positrons suffer different energy losses at high-, intermediate- and low-energies. Above a few tens of $\mathrm{GeV}$, inverse Compton and synchrotron radiation losses have the shortest timescales. Below a few MeV, ionisation and Coulomb losses dominate, and in-between, Bremsstrahlung losses dominate. However, some of the positrons measured below $10 \mathrm{GeV}$ have been produced at higher energy and at a more distant place. Hence, there is no one-to-one correspondence between the hierarchy in energy-loss timescales and impact on the positron flux. This motivates the detailed study of the impact of the various ingredient entering these losses.

Changing the interstellar radiation field (inverse Compton losses) and the magnetic fields (synchrotron losses) according to the values bracketed in Delahaye et al. (2010) have a $\sim 10 \%$ impact at $1 \mathrm{GeV}$. Coulomb losses on free electrons only dominate at very low energy, and we checked that the uncertainty on $n_{e}=0.033 \pm 0.002 \mathrm{~cm}^{-3}$ (Nordgren et al. 1992) - see Yao et al. (2017) for an updated model - has a negligible impact on the positron flux (sub-percent level) at $1 \mathrm{GeV}$.

Intermediate energies are dominated by Bremsstrahlung losses on the ISM gas. The same gas density is responsible for the production of secondary positrons. There is a further complication as this very gas density also directly impacts the determination of the transport coefficients, and this is discussed in the next paragraph. The gas density uncertainty is difficult to assess, and it can be probed for instance via the $\gamma$-ray emissivity (e.g. Delahaye et al. 2011), especially in the light of Fermi-LAT data (Ackermann et al. 2012; Casandjian 2015; Acero et al. 2016). The impact of the choice of different density maps was recently investigated in Jóhannesson et al. (2018): between 2D and 3D gas models, variations by a factor two on column density were found - this factor mostly comes from using an outdated value of the Sun's position in one gas component of the 2D model, and it certainly overestimates the uncertainty on the surface gas density $\left(\Sigma_{\mathrm{ISM}}\right)$. More realistically, the $H_{\mathrm{I}}$ spin temperature is already responsible for a $10 \%$ uncertainty in the gas density (Jóhannesson et al. 2018), and other sources of uncertainties come from the still debated $X_{\mathrm{CO}}$ conversion value (Remy et al. 2017) and the dark gas distribution (Grenier et al. 2005). For definiteness, we take a benchmark uncertainty of $50 \%$ on $\Sigma_{\text {ISM }}$ in the following. If we consider together the impact on the production and Bremsstrahlung, there should be no net effect in the regime where Bremsstrahlung losses dominate: The gas density cancels out from the integral calculation, as it appears both in the numerator (production) and in the denominator (propagation) - we stress however, that if primary electrons or positrons are considered (no production), their flux now scales with the inverse of the gas density, as found in Cirelli et al. (2013). We checked indeed that there is no impact of the gas density for secondary positrons at $1 \mathrm{GeV}$. However, at higher energies, in a regime where other energy losses dominate, we also find, as expected, a direct scaling with the gas density.

Fourthly, we consider uncertainties from transport coefficient calibration. The positron flux depends on the transport parameters, calibrated on secondary-to-primary ratios. For instance, assuming a know $\Sigma_{\text {ISM }}$, the parameter $K_{0}$ is determined with a $\sim \pm 12 \%$ uncertainty (Weinrich et al. 2020). This leads to a halved uncertainty on the positron flux at very low energy $\left(\propto 1 / \sqrt{K_{0}}\right)$, but it fully propagates at $1 \mathrm{GeV}$. However, as discussed in Maurin et al. (2010), any uncertainty on $\Sigma_{\text {ISM }}$ directly translates on the transport parameters $K_{0}, V_{\mathrm{c}}$, and $V_{\mathrm{A}}$, a behaviour also observed in Jóhannesson et al. (2018). A $\pm 50 \%$ change on $\Sigma_{\text {ISM }}$ would thus change $K_{0}$ (and $V_{\mathrm{A}}$ ) accordingly, which, combined with the impact on the positron production and Bremsstrahlung, leads to an overall $\pm 26 \%$ uncertainty on secondary positrons at $1 \mathrm{GeV}$, this number varying with the energy (see above).

Fifthly, we consider uncertainties from Solar modulation. Solar modulating the calculated positron flux also brings uncertainties. Above $100 \mathrm{GeV}$, CR fluxes are mostly unmodified, and TOA CRs below $1 \mathrm{GeV}$ mostly come from CRs at $\sim \mathrm{GeV}$. As a result, the error budget at $1 \mathrm{GeV}$ applies to lower energies as well for TOA positrons.

Modulation levels for most data in this analysis were taken from Ghelfi et al. (2017b), that is, from averages - over the appropriate CR data taking periods - of time series based on the analysis of neutron monitor data. The fact that post-fit values in the LiBeB analyses (see the companion paper, Weinrich et al. 2020) were found to be consistent with the above assumed values further support this choice. In practice, however, Ghelfi et al. (2017b) time series do not extend after 2017. To derive the positron modulation level $\phi_{\mathrm{FF}}=650 \mathrm{MV}$, we relied on Oulu times series ${ }^{6}$, derived from Usoskin et al. (2005), and rescaled according to $\phi_{\text {Ghelfi17 }}(t) \approx \phi_{\text {Usoskin05 }}(t)+100 \mathrm{MV}$, as found in Ghelfi et al. (2017b). The overall uncertainties on reconstructed modulation levels from neutron monitors are $\pm 100 \mathrm{MV}$ (Ghelfi et al. 2017b). Those obtained from directly fitting TOA (preAMS) $\mathrm{H}$ and $\mathrm{He}$ data are in the $\pm 30 \mathrm{MV}$ range (Ghelfi et al. 2016). We choose a very conservative approach below, and for $1 \mathrm{GeV}$ secondary positrons, the $\pm 100 \mathrm{MV}( \pm 15 \%)$ uncertainty translates into a $\mp 50 \%$ uncertainty. This makes modulation the dominant source of uncertainty for the positron flux calculation.

To conclude this section, we summarise our results on the uncertainties. Although the above analysis does not reach the level of refinement developed for CR nuclei analyses (Derome et al. 2019), we now have a quantitative grasp on the uncertainties on the secondary positron calculations. They are gathered in Table 4, where we also provide a finer view of these uncertainties at three energies $(10 \mathrm{MeV}, 1 \mathrm{GeV}$, and $100 \mathrm{GeV})$. At $1 \mathrm{GeV}$, which is the energy that is relevant for the analysis below, uncertainties from Solar modulation are the dominant effect, followed by those on production and $\Sigma_{\text {ISM }}$. Regarding production, we nevertheless stress that the assumptions we make are conservative

6 http://cosmicrays.oulu.fi/phi 
Table 4. Error budget on the calculation of secondary positrons.

\begin{tabular}{|c|c|c|}
\hline Ingredient & $\begin{array}{c}\text { Error on } \\
\text { ingredient [\%] }\end{array}$ & $\begin{array}{c}\Delta \Phi_{\mathrm{e}^{+}}^{\mathrm{IS}} / \Phi_{\mathrm{e}^{+}}^{\mathrm{IS}}[\%] \\
\text { at }\left(10^{-2}, 1,10^{2}\right) \mathrm{GeV}^{(\star)}\end{array}$ \\
\hline \multicolumn{3}{|c|}{$\mathrm{CR}$ and gas composition } \\
\hline $\mathrm{CR} \mathrm{H}$ and $\mathrm{He}$ & $\pm 10 \%$ & $\pm 10 \%$ \\
\hline$+\operatorname{CRs}(Z>2)$ & - & $+10 \%$ \\
\hline$+\operatorname{ISM}(Z>2)$ & - & $\pm 3 \%$ \\
\hline \multicolumn{3}{|l|}{ Energy losses } \\
\hline ISRF & $\# 1 \rightarrow \# 2^{(\dagger)}$ & $(+0.2 \%,-2.7 \%,-4.1 \%)$ \\
\hline B & $\pm 1 \mu \mathrm{G}$ & $( \pm 0.7 \%, \mp 9.5 \%, \mp 12 \%)$ \\
\hline$n_{e}$ & $\pm 10 \%$ & $(\mp 0.2 \%, \mp 0.7 \%,<0.1 \%)$ \\
\hline \multicolumn{3}{|c|}{ Transport calibration and positron production } \\
\hline $\begin{array}{l}K_{0} \& V_{\mathrm{A}} \\
(\mathrm{d} \sigma / \mathrm{d} E)^{\mathrm{prod}}\end{array}$ & $\begin{array}{l} \pm 12 \% \\
+20 \%\end{array}$ & $\begin{array}{cl}(\mp 7 \%, & \pm 12 \%, \quad \mp 5 \%) \\
& +20 \%\end{array}$ \\
\hline \multicolumn{3}{|c|}{ Surface density $\left(\boldsymbol{\Sigma}_{\text {ISM }}\right)$} \\
\hline$\Sigma_{\text {ISM }}^{\mathrm{PB} \equiv \text { Prod. \& Brem. }}$ & $\pm 50 \%$ & $( \pm 13 \%, \pm 0.7 \%, \pm 49 \%)$ \\
\hline$\sum_{\mathrm{ISM}}^{\mathrm{All}}\left(\mathrm{PB} \& \mathrm{~K}_{0} \& \mathrm{~V}_{\mathrm{A}}\right)$ & $\pm 50 \%$ & $(\mp 0.7 \%, \pm 26 \%, \pm 21 \%)$ \\
\hline \multicolumn{2}{|c|}{ Solar modulation (TOA fluxes) } & $\Delta \Phi_{e^{+}}^{\mathrm{TOA}} / \Phi_{e^{+}}^{\mathrm{TOA}}[\%]$ \\
\hline$\phi$ & $\pm 15 \%$ & $\left(\mathrm{n} / \mathrm{a}^{(\ddagger)}, \quad \mp 50 \%,<1 \%\right)$ \\
\hline
\end{tabular}

Notes. The first column list the quantities varied in the calculation, the second column provide the typical uncertainties on this ingredient, and the last three columns show the corresponding uncertainty on the calculated IS secondary flux of positrons-the gas surface density impacts the calculation in different places, and its impact is broken down below (see text for details). A "+" sign below (instead of " \pm ") means that our calculation is conservative, that is, the secondary flux would be larger if we were to account for these specific ingredients. The exact numbers slightly depend on the configuration used (BIG, SLIM, or QUAINT) and we report below values from QUAINT. ${ }^{(\star)}$ For energy-dependent effects, we report 3 values in the table, otherwise a single value is provided. ${ }^{(\dagger)}$ Two parametrisations taken from Delahaye et al. (2010). ${ }^{\left({ }^{(*)}\right.}$ At very low energy, the TOA flux is strongly suppressed and its variation is not very meaningful to report.

to derive lower limits on $L$; they underestimate the positron flux by $\sim 20-30 \%$ (10-20\% from cross sections and $10 \%$ from unaccounted for heavy CR projectiles).

\subsubsection{Constraints on $L$}

To check the compatibility with the limits set by AMS-02 Be/B data, we carried out calculations for several $L$ values. The latter are taken inside their allowed range (upper-half of Table 3). To ensure a consistent calculation, the transport parameters are rescaled with $L$ according to Eq. (A.1) and values in Table A.1 (see Appendix A).

We set conservative limits on $L$ by excluding propagation setups for which the prediction of the secondary positron flux exceeds the AMS-02 measurements (Aguilar et al. 2019a) by more than $3 \sigma$ in a single bin. In practice, for all our configurations, the lowest-energy AMS-02 data point is the one setting constraints.

In a first step, we check the consistency of the positron flux with the $\mathrm{Be} / \mathrm{B}$ constraint. In Fig. 5, we report our predictions for the secondary positron fluxes (rescaled by $E^{3}$ ) for the BIG, SLIM, and QUAINT propagation models (left, middle, and right panel, respectively) along with the AMS-02 data (Aguilar et al. 2019a). These predictions are derived using the best-fit parameters inferred from the combined $\mathrm{Li} / \mathrm{C}, \mathrm{Be} / \mathrm{C}$, and $\mathrm{B} / \mathrm{C}$ analysis performed in Weinrich et al. (2020), already extensively discussed throughout the paper. For illustration, we have taken the best-fit values obtained for $L$ from the AMS-02 Be/B data only (see Table 3 ). In each panel, the solid curve corresponds to the best-fit value for $L$, bracketed by its $1 \sigma$ statistical uncertainty (dashed curve for the lower deviation, and dot-dashed curve for the upper deviation).

From Fig. 5, we see that the positron constraint on $L$ is only relevant for the QUAINT propagation model. The latter has an effective Alfvèn speed $V_{\mathrm{A}} \sim 40 \mathrm{~km} \mathrm{~s}^{-1}$ (see Table A.1) which sets the amplitude of reacceleration ${ }^{7}$. For the BIG and SLIM models (left and middle panels), both devoid of reacceleration, the nominal predictions undershoot the data. The low-energy behaviour in these models is furthermore exacerbated by the low-rigidity break in the diffusion coefficient around 3-4 GV (Weinrich et al. 2020): it leads to a lower secondary positron flux at low rigidities and hence a decreased constraining power on $L$. Given the uncertainties on the calculation, only QUAINT can overshoot the data, whereas BIG and SLIM cannot for $L$ values constrained by $\mathrm{Be} / \mathrm{B}$.

In a second step, we derive the upper-limit on $L$ in the model QUAINT. Our nominal $3 \sigma$ constraint for QUAINT is $L \geq 5.54 \mathrm{kpc}$. However, this number is very sensitive to the model uncertainties. For illustration, we show in Fig. 6 the impact of Solar modulation uncertainties, varying the Fisk potential by a generous $\pm 100 \mathrm{MV}$ (Ghelfi et al. 2017b). The upper value of the modulation, which minimises the modulated positron flux, should be viewed as conservative, weakening the limit to $L \geq 2.9 \mathrm{kpc}$. The lower value corresponds to a more aggressive constraint (for a lower solar activity) $L \geq 11.3 \mathrm{kpc}$. These limits do not account for sub-leading uncertainties discussed above. Without a better handle on the uncertainties, it is difficult to firmly conclude. Overall, we note that the positron constraint on $L$ for the QUAINT model is in agreement with those derived from the $\mathrm{Be} / \mathrm{B}$ and ${ }^{10} \mathrm{Be} /{ }^{9} \mathrm{Be}$ analysis, while being independent from the latter.

We have only discussed the case of overshooting, which provides a clear situation for excluding part of the parameter space. Given that in the BIG and SLIM models the nominal predictions undershoot the data, one may wonder if their viability is questioned by the positron data. We remark that the various sources of uncertainties (modulation, production, surface gas density), estimated at the $\$ 50 \%$ level at most, seem unable to fully account for the factor of $\sim 2$ mismatch. However, primary positrons, already necessary to explain the high-energy positron fraction in pre-AMS-02 studies, might make up a significant fraction of the positron budget also at low energy - for example, the absence of primary positrons at low energy was raised as an important issue for astrophysical sources like pulsar wind nebulae in Blasi \& Amato (2011). Therefore, discussing further the consistency of any propagation model (not only BIG and SLIM) with the positron data should rely on analyses also including primary positrons. This goes beyond the scope of this paper.

\section{Conclusions}

In the context of recent high-precision AMS-02 data, we have revisited the constraints set on the halo size of the Galaxy from radioactive species and positron fluxes.

Using AMS-02 Be/B data we find $L=5_{-2}^{+3} \mathrm{kpc}$ at $1 \sigma$, in agreement with Boschini et al. (2020) (but with a larger error)

\footnotetext{
7 We stress that pre-AMS-02 fit to B/C data (e.g. Maurin et al. 2001) had larger $V_{\mathrm{A}}$. It was found that strong reacceleration gave rise to a prominent bump around $1 \mathrm{GeV}$ in the predicted positron flux, which then easily overshoots the data, especially below a few GeV (Delahaye et al. 2009; Lavalle et al. 2014; Boudaud et al. 2017). However, with milder reacceleration here, we see in the right panel that the flux predictions associated with QUAINT are not in that strong excess with respect to the data, and do not feature any significant bump.
} 

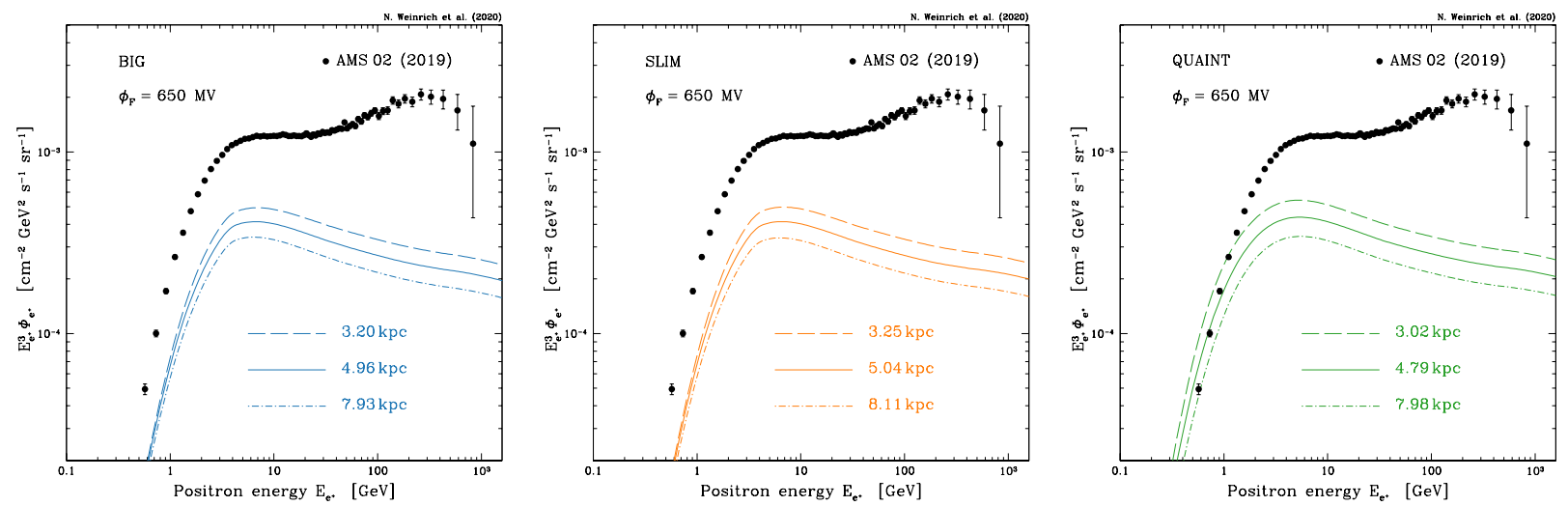

Fig. 5. Secondary positron flux predictions using the best-fit transport parameters of the BIG (left panel), SLIM (middle panel), and QUAINT (right panel) propagation models, as inferred from the AMS- 02 combined $\mathrm{Li} / \mathrm{C}, \mathrm{Be} / \mathrm{B}$, and $\mathrm{B} / \mathrm{C}$ analysis. The three lines correspond to TOA fluxes, modulated at $650 \mathrm{MV}$, for the best-fit (solid line) and $1 \sigma$ upper (dash-dotted) and lower (dashed) limits on $L$ (see Table 3) - AMS-02 data are from Aguilar et al. (2019a).

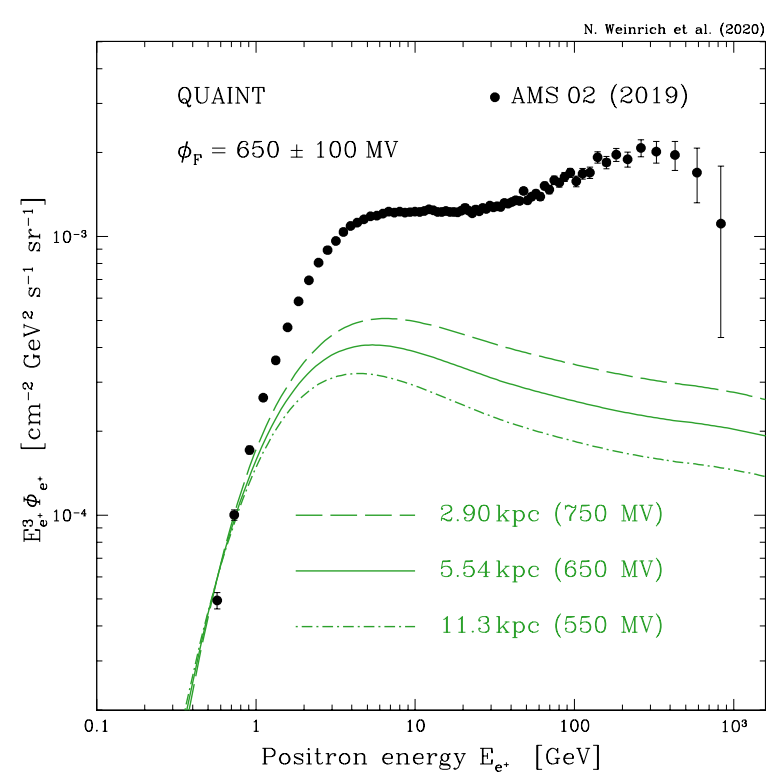

Fig. 6. Secondary positron flux predictions using the best-fit transport parameters of the QUAINT propagation model (also inferred from the AMS-02 combined $\mathrm{Li} / \mathrm{C}, \mathrm{Be} / \mathrm{B}$, and $\mathrm{B} / \mathrm{C}$ analysis). We show the predictions associated with the $3 \sigma$ limits on $L$ assuming different solar modulation strengths.

and less than one sigma away from the results of Evoli et al. (2020). Our result holds for several transport configurations (Derome et al. 2019; Weinrich et al. 2020), namely BIG (reacceleration and low-rigidity break), SLIM (pure diffusion with low-rigidity break), and QUAINT (reacceleration and diffusion upturn in the non-relativistic regime). The constraints are tighter (factor $\sim 2$ reduction in errors) and move to a lower value of $L$ (by $0.3-0.7 \mathrm{kpc}$ ) when low-energy ${ }^{10} \mathrm{Be} /{ }^{9} \mathrm{Be}$ data are considered, but this tightening may be related to the fact that ISOMAX data (Hams et al. 2004) prefer a smaller halo size $L \approx 3 \mathrm{kpc}$ and might be indicative of a tension in the data. With the recent release of ${ }^{3} \mathrm{He}$ and ${ }^{4} \mathrm{He}$ data (Aguilar et al. 2019b), AMS-02 demonstrated its capabilities for measuring isotopic fluxes. Separating Be isotopes will certainly be even more challenging, but AMS02 will provide a unique picture in the few $\mathrm{GeV} / \mathrm{n}$ regime, where only ISOMAX and preliminary PAMELA data (Bogomolov \& Vasilyev 2019) are available for now, and shed some light on the mutual consistency of these datasets. The balloon-borne HELIX, should also provide a complementary view. The instru- ment is scheduled for a long-duration balloon flight in 2020/21, and is expected to achieve a $10 \%$ statistical error on ${ }^{10} \mathrm{Be} /{ }^{9} \mathrm{Be}$ in the $0.1-10 \mathrm{GeV} / \mathrm{n}$ range (Park et al. 2019).

We have also performed a detailed analysis of the modelling uncertainties for both the $\mathrm{Be} / \mathrm{B}$ and ${ }^{10} \mathrm{Be} /{ }^{9} \mathrm{Be}$ ratios to understand whether better data could help improving the estimation on $L$ in the future. Whereas $\mathrm{Be} / \mathrm{B}$ is maximally sensitive to $L$ at a few tens of $\mathrm{GV},{ }^{10} \mathrm{Be} /{ }^{9} \mathrm{Be}$ is sensitive to $L$ over a much larger range (from $100 \mathrm{MeV} / \mathrm{n}$ to tens of $\mathrm{GeV} / \mathrm{n}$ ). In terms of possible improvements, $\mathrm{Be} / \mathrm{B}$ data are already limited by systematics, whereas this is not yet the case for ${ }^{10} \mathrm{Be} /{ }^{9} \mathrm{Be}$. For these reasons, ${ }^{10} \mathrm{Be} /{ }^{9} \mathrm{Be}$ seems to be the best target for future experiments. However, in both cases, production crosssections uncertainties dominate the modelling error budget and are already at the level of data uncertainties. Future improvements on $L$ will thus not be possible without improving nuclear data. An alternative strategy to mitigate these uncertainties would be to combine data from different $\mathrm{CR}$ clocks (e.g. $\mathrm{Al} / \mathrm{Mg}$ and $\mathrm{Cl} / \mathrm{Ar}$ ).

In a broader context of multi-wavelength and multimessenger observations, we also discussed the constraints set by synchrotron radio (e.g. Di Bernardo et al. 2013) and diffuse $\gamma$-ray emissions (e.g. Tibaldo et al. 2015) in the Milky-way (and in some cases in other galaxies). The various observations lead to lower or upper limits, defining a broad range $L \in[2,10] \mathrm{kpc}$ compatible with results from radioactive CR nuclei. We also updated the constraints set by positrons (Lavalle et al. 2014). Since BIG and SLIM configurations undershoot the data, the constraints are only significant for the QUAINT model, leading to $L \geq 2.9-11.3 \mathrm{kpc}$ depending on the solar modulation, with a nominal central value $L \geq 5.5 \mathrm{kpc}$; these numbers could shift upwards or downwards depending on uncertainties on the production cross section and the gas surface density. Within the errors, these constraints are also consistent with the ones derived in the main analysis.

While our conclusions appear rather robust within the framework of this analysis, one should keep in mind that these bounds might be altered in presence of inhomogeneities in the local gas density (for radioactive species) and on the diffusion coefficient (for both radioactive species and positrons). These extensions represent interesting and motivated subjects for future studies.

Acknowledgements. We thank A. Marcowith for discussions. This work has been supported by Univ. de Savoie, appel à projets: Diffusion from Galactic High-Energy Sources to the Earth (DIGHESE), by the Programme National 
des Hautes Energies of CNRS/INSU with INP and IN2P3, co-funded by CEA and CNES, and by Villum Fonden under project no. 18994. This work is partly supported by the ANR project ANR-18-CE31-0006, the national CNRS-INSU programmes PNHE and PNCG, and European Union's Horizon 2020 research and innovation programme under the Marie Skłodowska-Curie grant agreements $\mathrm{N}^{\circ} 690575$ and $\mathrm{N}^{\circ} 674896$.

\section{References}

Abeysekara, A. U., Albert, A., Alfaro, R., et al. 2017, Science, 358, 911 Acero, F., Ackermann, M., Ajello, M., et al. 2016, ApJS, 223, 26 Ackermann, M., Ajello, M., Atwood, W. B., et al. 2012, ApJ, 750, 3 Adriani, O., Ambrosi, G., Baoudoy, B., et al. 2019, White Paper submitted in response to ESA's Call for the VOYAGE 2050 long-term plan

Adriani, O., Barbarino, G. C., Bazilevskaya, G. A., et al. 2009, Nature, 458, 607 Aguilar, M., Alcaraz, J., Allaby, J., et al. 2011, ApJ, 736, 105

Aguilar, M., Aisa, D., Alpat, B., et al. 2015, Phys. Rev. Lett., 114, 171103

Aguilar, M., Ali Cavasonza, L., Alpat, B., et al. 2017, Phys. Rev. Lett., 119 251101

Aguilar, M., Ali Cavasonza, L., Ambrosi, G., et al. 2018, Phys. Rev. Lett., 120, 021101

Aguilar, M., Ali Cavasonza, L., Ambrosi, G., et al. 2019a, Phys. Rev. Lett., 122, 041102

Aguilar, M., Ali Cavasonza, L., Ambrosi, G., et al. 2019b, Phys. Rev. Lett., 123, 181102

Aharonian, F. A., Atoyan, A. M., \& Voelk, H. J. 1995, A\&A, 294, L41

Aramaki, T., Boggs, S., Bufalino, S., et al. 2016, Phys. Rep., 618, 1

Beck, R. 2015, A\&ARv, 24, 4

Bergström, L., Bringmann, T., \& Edsjö, J. 2008, Phys. Rev. D, 78, 103520

Biswas, S., \& Gupta, N. 2018, J. Cosmol. Astropart. Phys., 2018, 063

Blasi, P., \& Amato, E. 2011, Astrophys. Space Sci. Proc., 21, 624

Blasi, P., Amato, E., \& Serpico, P. D. 2012, Phys. Rev. Lett., 109, 061101

Bogomolov, E. A., \& Vasilyev, G. I. 2019, Bull. Russ. Acad. Sci. Phys., 83, 967

Boschini, M. J., Torre, S. D., Gervasi, M., et al. 2020, ApJ, 889, 167

Boudaud, M., Bueno, E. F., Caroff, S., et al. 2017, A\&A, 605, A17

Boudaud, M., Génolini, Y., Derome, L., et al. 2020, Phys. Rev. Res., 2, 023022

Breitschwerdt, D., McKenzie, J. F., \& Voelk, H. J. 1991, A\&A, 245, 79

Bringmann, T., Donato, F., \& Lineros, R. A. 2012, J. Cosmol. Astropart. Phys., 2012, 049

Bulanov, S. V., Syrovatskii, C. I., \& Dogel, V. A. 1976, Ap\&SS, 44, 255

Caballero-Lopez, R. A., \& Moraal, H. 2004, JGR, 109, 1101

Casandjian, J.-M. 2015, ApJ, 806, 240

Cattaneo, P. W., \& HERD Collaboration 2019, Nucl. Part. Phys. Proc., 306, 85

Cholis, I., Linden, T., \& Hooper, D. 2019, Phys. Rev. D, 99, 103026

Cirelli, M., Serpico, P. D., \& Zaharijas, G. 2013, JCAP, 1311, 035

Connell, J. J. 1998, ApJ, 501, L59

Conrad, J., \& Reimer, O. 2017, Nat. Phys., 13, 224

Cuoco, A., Heisig, J., Klamt, L., Korsmeier, M., \& Krämer, M. 2019, Phys. Rev. D, 99, 103014

de Nolfo, G. A., Moskalenko, I. V., Binns, W. R., et al. 2006, AdSR, 38, 1558

Delahaye, T., Fiasson, A., Pohl, M., \& Salati, P. 2011, A\&A, 531, A37

Delahaye, T., Lineros, R., Donato, F., Fornengo, N., \& Salati, P. 2008, Phys Rev. D, 77, 063527

Delahaye, T., Lineros, R., Donato, F., et al. 2009, A\&A, 501, 821

Delahaye, T., Lavalle, J., Lineros, R., Donato, F., \& Fornengo, N. 2010, A\&A, 524, A51

Derome, L., Maurin, D., Salati, P., et al. 2019, A\&A, 627, A158

Di Bernardo, G., Evoli, C., Gaggero, D., Grasso, D., \& Maccione, L. 2010 Astropart. Phys., 34, 274

Di Bernardo, G., Evoli, C., Gaggero, D., Grasso, D., \& Maccione, L. 2013, J Cosmol. Astropart. Phys., 2013, 036

Di Mauro, M., Manconi, S., \& Donato, F. 2019, Phys. Rev. D, 100, 123015

Donato, F., Fornengo, N., Maurin, D., Salati, P., \& Taillet, R. 2004, Phys. Rev. D, 69, 063501

Donato, F., Maurin, D., \& Taillet, R. 2002, A\&A, 381, 539

Duvernois, M. A., Simpson, J. A., \& Thayer, M. R. 1996, A\&A, 316, 555

Evoli, C., Gaggero, D., Grasso, D., \& Maccione, L. 2012, Phys. Rev. Lett., 108, 211102

Evoli, C., Blasi, P., Morlino, G., \& Aloisio, R. 2018, Phys. Rev. Lett., 121, 021102

Evoli, C., Aloisio, R., \& Blasi, P. 2019, Phys. Rev. D, 99, 103023

Evoli, C., Morlino, G., Blasi, P., \& Aloisio, R. 2020, Phys. Rev. D, 101, 023013

Feng, J., \& Zhang, H.-H. 2018, ApJ, 858, 116

Fields, B., Ellis, J. R., Binns, W. R., et al. 2019, BAAS, 51, 410

Garcia-Munoz, M., Mason, G. M., \& Simpson, J. A. 1977, ApJ, 217, 859

Garcia-Munoz, M., Simpson, J. A., \& Wefel, J. P. 1981, Int. Cosmic Ray Conf., 2,72
Garcia-Munoz, M., Simpson, J. A., Guzik, T. G., Wefel, J. P., \& Margolis, S. H. 1987, ApJS, 64, 269

Génolini, Y., Serpico, P. D., Boudaud, M., et al. 2017, Phys. Rev. Lett., 119, 241101

Génolini, Y., Boudaud, M., Batista, P. I., et al. 2019, Phys. Rev. D, 99, 123028

Ghelfi, A., Barao, F., Derome, L., \& Maurin, D. 2016, A\&A, 591, A94

Ghelfi, A., Barao, F., Derome, L., \& Maurin, D. 2017a, A\&A, 605, C2

Ghelfi, A., Maurin, D., Cheminet, A., et al. 2017b, AdSR, 60, 833

Girichidis, P., Pfrommer, C., Hanasz, M., \& Naab, T. 2020, MNRAS, 491, 993

Gleeson, L. J., \& Axford, W. I. 1967, ApJS, 149, L115

Gleeson, L. J., \& Axford, W. I. 1968, ApJ, 154, 1011

Grenier, I. A., Casandjian, J.-M., \& Terrier, R. 2005, Science, 307, 1292

Hams, T., Barbier, L. M., Bremerich, M., et al. 2004, ApJ, 611, 892

Hooper, D., Blasi, P., \& Dario Serpico, P. 2009, J. Cosmol. Astropart. Phys., 1, 25

James, F., \& Roos, M. 1975, Comput. Phys. Comm., 10, 343

Jóhannesson, G., Ruiz de Austri, R., Vincent, A. C., et al. 2016, ApJ, 824, 16

Jóhannesson, G., Porter, T. A., \& Moskalenko, I. V. 2018, ApJ, 856, 45

Jones, F. C., Lukasiak, A., Ptuskin, V., \& Webber, W. 2001, ApJ, 547, 264

Kachelrieß, M., Moskalenko, I. V., \& Ostapchenko, S. 2019, Comput. Phys. Commun., 245, 106846

Kamae, T., Karlsson, N., Mizuno, T., Abe, T., \& Koi, T. 2006, ApJ, 647, 692

Krause, M. 2014, ArXiv e-prints [arXiv:1401.1317]

Krombel, K. E., \& Wiedenbeck, M. E. 1988, ApJ, 328, 940

Lavalle, J., Maurin, D., \& Putze, A. 2014, Phys. Rev. D, 90, 081301

Lave, K. A., Wiedenbeck, M. E., Binns, W. R., et al. 2013, ApJ, 770, 117

Lukasiak, A. 1999, Int. Cosmic Ray Conf., 3, 41

Manconi, S., Di Mauro, M., \& Donato, F. 2019, J. Cosmol. Astropart. Phys., 2019,024

Maurin, D. 2020, Comput. Phys. Commun., 247, 106942

Maurin, D., \& Taillet, R. 2003, A\&A, 404, 949

Maurin, D., Donato, F., Taillet, R., \& Salati, P. 2001, ApJ, 555, 585

Maurin, D., Putze, A., \& Derome, L. 2010, A\&A, 516, A67

Maurin, D., Melot, F., \& Taillet, R. 2014, A\&A, 569, A32

Maurin, D., Cheminet, A., Derome, L., Ghelfi, A., \& Hubert, G. 2015, AdSR, 55,363

Menn, W., Bogomolov, E. A., Simon, M., et al. 2018, ApJ, 862, 141

Moskalenko, I. V., Mashnik, S. G., \& Strong, A. W. 2001, ICRC, 5, 1836

Nordgren, T. E., Cordes, J. M., \& Terzian, Y. 1992, AJ, 104, 1465

Orlando, E., \& Strong, A. 2013, MNRAS, 436, 2127

Park, N., Beaufore, L., Mbarek, R., et al. 2019, Int. Cosmic Ray Conf., 36, 121

Profumo, S., Reynoso-Cordova, J., Kaaz, N., \& Silverman, M. 2018, Phys. Rev. D, 97, 123008

Ptuskin, V. S., Moskalenko, I. V., Jones, F. C., Strong, A. W., \& Zirakashvili, V. N. 2006, ApJ, 642, 902

Putze, A., Derome, L., \& Maurin, D. 2010, A\&A, 516, A66

Reinert, A., \& Winkler, M. W. 2018, J. Cosmology Astropart. Phys., 2018, 055

Remy, Q., Grenier, I. A., Marshall, D. J., \& Casand jian, J. M. 2017, A\&A, 601, A78

Schael, S., Atanasyan, A., Berdugo, J., et al. 2019, Nucl. Inst. Methods Phys. Res. A, 944, 162561

Schmidt, P., Krause, M., Heesen, V., et al. 2019, A\&A, 632, A12

Seo, E. S., McDonald, F. B., Lal, N., \& Webber, W. R. 1994, ApJ, 432, 656

Serpico, P. D. 2012, Astropart. Phys., 39, 2

Stecker, F. W., \& Jones, F. C. 1977, ApJ, 217, 843

Stone, E. C., Cummings, A. C., McDonald, F. B., et al. 2013, Science, 341, 150

Taillet, R., \& Maurin, D. 2003, A\&A, 402, 971

Tibaldo, L., Digel, S. W., Casandjian, J. M., et al. 2015, ApJ, 807, 161

Tomassetti, N. 2015a, Phys. Rev. C, 92, 045808

Tomassetti, N. 2015b, Phys. Rev. D, 92, 081301

Trotta, R., Jóhannesson, G., Moskalenko, I. V., et al. 2011, ApJ, 729, 106

Usoskin, I. G., Alanko-Huotari, K., Kovaltsov, G. A., \& Mursula, K. 2005, JGR, 110,12108

Vittino, A., Mertsch, P., Gast, H., \& Schael, S. 2019, Phys. Rev. D, 100, 043007 Webber, W. R. 1982, ApJ, 252, 386

Webber, W. R., Lezniak, J. A., Kish, J., \& Damle, S. V. 1973, Ap\&SS, 24, 17

Webber, W. R., Kish, J., \& Simpson, G. 1979, ICRC, 1, 430

Webber, W. R., \& Soutoul, A. 1998, ApJ, 506, 335

Weinrich, N., Génolini, Y., Boudaud, M., Derome, L., \& Maurin, D. 2020, A\&A, in press, https://doi.org/10.1051/0004-6361/202037875

Wiedenbeck, M. E., \& Greiner, D. E. 1980, ApJ, 239, L139

Yanasak, N. E., Wiedenbeck, M. E., Mewaldt, R. A., et al. 2001, ApJ, 563, 768

Yao, J. M., Manchester, R. N., \& Wang, N. 2017, ApJ, 835, 29

Young, J. S., Freier, P. S., Waddington, C. J., Brewster, N. R., \& Fickle, R. K. 1981, ApJ, 246, 1014

Zirakashvili, V. N., Breitschwerdt, D., Ptuskin, V. S., \& Voelk, H. J. 1996, A\&A, 311,113 


\section{Appendix A: Scaling relations with $L$}
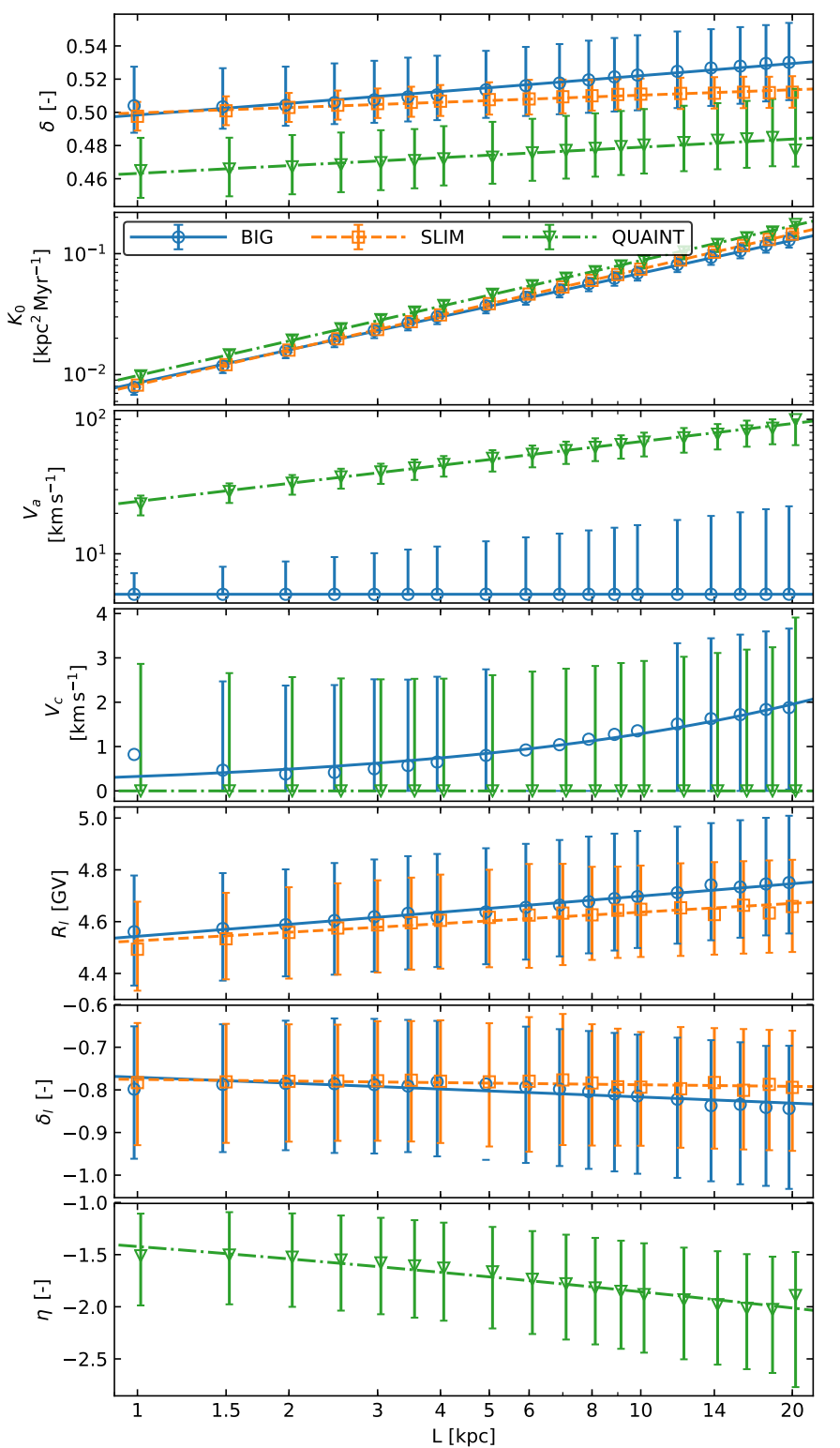

Fig. A.1. Transport parameters dependence on the halo size $L$. Colourcoded symbols correspond to the best-fit values on the combined analysis of $\mathrm{Li} / \mathrm{C}, \mathrm{Be} / \mathrm{B}$, and $\mathrm{B} / \mathrm{C}$ data for different models (BIG, SLIM, and QUAINT). The lines show, for each model and transport parameter, power-law fits to these data whose values are reported in the right-hand side of Table A.1.

In Sect. 3.2, we study the sensitivity to $L$ of $C R$ data combinations involving a radioactive species. To do so, the transport parameters obtained from the study of $\mathrm{B} / \mathrm{C}$ must be known for any $L$. In Sect. 4.2 , we test whether secondary positrons overshoot the data given for various $L$, given the constraints set from secondary-to-primary ratios and radioactive species. This is a similar but slightly different question than the previous one.

For each model (BIG, SLIM, and QUAINT), we repeat the transport parameter fitting procedure described in Weinrich et al. (2020) at fixed $L$ for several values of $L$, for either the AMS-02 $\mathrm{B} / \mathrm{C}$ ratio only, or the combined AMS- $02 \mathrm{Li} / \mathrm{C}, \mathrm{Be} / \mathrm{B}$, and $\mathrm{B} / \mathrm{C}$ data. We show for the latter the resulting best-fit transport parameters (symbols) in Fig. A.1. We find that a simple power law is

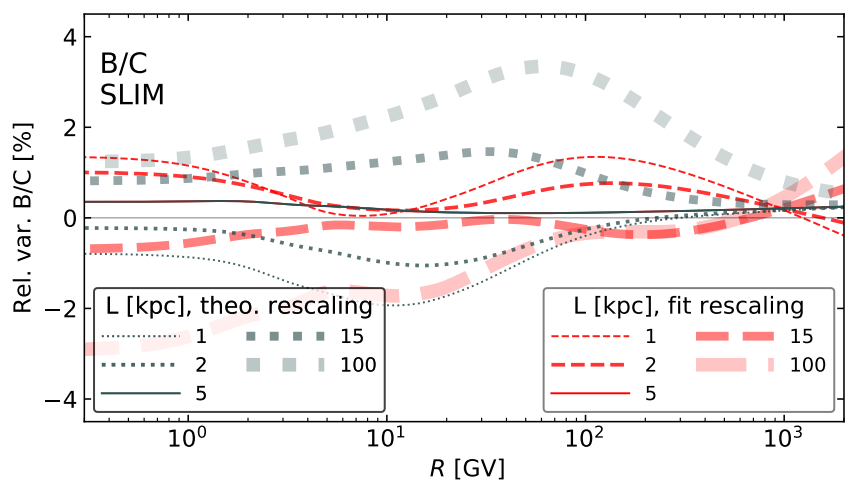

Fig. A.2. Relative variation of $\mathrm{B} / \mathrm{C}$ ratio (with respect to a reference taken at $L=5 \mathrm{kpc}$ ), as a function of rigidity. Thin- to thick-dashed lines correspond to calculations for various $L$ using either (i) the "theoretical" rescaling which enforce the same level of production for stable secondary species (grey lines), or (ii) the rescaling based on a refit of $\mathrm{B} / \mathrm{C}$ data for each $L$ as described in the text (red lines).

enough to capture the dependence with $L$ (lines in Fig. A.1),

$\operatorname{Param}(L)=A \cdot\left(\frac{L}{5 \mathrm{kpc}}\right)^{B}$,

and we report the best-fit $A$ and $B$ values found for each transport parameter $\left(\delta, K_{0}\right.$, etc. $)$ in Table A.1 for the two different cases.

\section{A.1. Power-law behaviour of the rescaling}

A few comments are in order: the power-law indices, $B$, for the $\mathrm{B} / \mathrm{C}$ analysis are very similar to the ones found in Fig. 5 of Putze et al. (2010), that is 1.06 for $K_{0}$ and 0.53 for $V_{\mathrm{A}}$ (from the analysis of older B/C data). As argued in Putze et al. (2010), a secondary-to-primary ratio provides constraints degenerated with $K_{0} / L$ and $V_{\mathrm{A}}^{2} / L$ ratios. These small differences (1.06 vs. 1 and 0.53 vs. 0.5$)$ originate in the small fraction of ${ }^{10} \mathrm{~B}(\lesssim 10 \%)$ coming from decayed ${ }^{10} \mathrm{Be}$, which is sensitive to $L$. Figure A.2 illustrates this sensitivity on the $\mathrm{B} / \mathrm{C}$ ratio. If the theoretical rescaling is applied (grey lines), the curves with varying $L$ are either above or below the reference, converging to the reference at high rigidity as ${ }^{10} \mathrm{Be}$ half-life grows and ${ }^{10} \mathrm{Be}$ behaves as a stable secondary species. At variance, when the above rescaling is applied, the variation with $L$ is absorbed in the fit, and the highrigidity limit no longer goes to zero. The overall difference is at the few percent level.

\section{A.2. Difference between $B / C$ and combined analysis results}

A similar scaling remains for the combined analysis $(\mathrm{Li} / \mathrm{C}, \mathrm{Be} / \mathrm{B}$, $\mathrm{B} / \mathrm{C})$, but with some differences, that were all highlighted in Weinrich et al. (2020). First, the diffusion coefficient normalisation $K_{0}$ is smaller than in the $\mathrm{B} / \mathrm{C}$ only case, and the difference is related to partial degeneracies with production cross sections, that are lifted in the combined analysis of elements. The bestfit values are consistent, though slightly different, from those in Weinrich et al. (2020), and this is mostly attributed to the fact that we fit here the combination $\mathrm{Be} / \mathrm{B}$ instead of $\mathrm{Be} / \mathrm{C}$. Second, whereas for the $\mathrm{B} / \mathrm{C}$ case $\mathrm{BIG}$ parameters are very similar to QUAINT ones, for the combined analysis the BIG parameters are closer to SLIM ones. Last, the low-energy parameters $\delta_{l}$ and $\eta$ are also different from the $\mathrm{B} / \mathrm{C}$ analysis only, and correspond to more marked low-rigidity break of the diffusion coefficient; we find here that the latter parameters have almost no dependence on $L$. 
Table A.1. Scaling coefficients for the transport parameters, see Eq. (A.1), with $B$ the power-law slope and $A$ the transport parameter value for $L=5 \mathrm{kpc}$.

\begin{tabular}{|c|c|c|c|c|c|c|c|}
\hline \multirow[t]{2}{*}{ Parameter } & \multirow[t]{2}{*}{ Coef. } & \multicolumn{3}{|c|}{ Fit $\mathrm{B} / \mathrm{C}$} & \multicolumn{3}{|c|}{ Fit $\mathrm{Li} / \mathrm{C}, \mathrm{Be} / \mathrm{B}$, and $\mathrm{B} / \mathrm{C}$} \\
\hline & & $\mathrm{BIG}$ & SLIM & QUAINT & $\mathrm{BIG}$ & SLIM & QUAINT \\
\hline \multirow[t]{2}{*}{$\delta[-]$} & A & 0.488 & 0.511 & 0.458 & 0.515 & 0.507 & 0.474 \\
\hline & B & -0.013 & -0.011 & -0.013 & 0.020 & 0.009 & 0.015 \\
\hline \multirow{2}{*}{$K_{0}\left[\mathrm{kpc}^{2} \mathrm{Myr}^{-1}\right]$} & A & 0.048 & 0.043 & 0.056 & 0.037 & 0.038 & 0.045 \\
\hline & B & 1.043 & 1.034 & 1.040 & 0.907 & 0.957 & 0.952 \\
\hline \multirow{2}{*}{$V_{\mathrm{A}}\left[\mathrm{km} \mathrm{s}^{-1}\right]$} & A & 42.94 & $\mathrm{n} / \mathrm{a}$ & 67.24 & 5.001 & $\mathrm{n} / \mathrm{a}$ & 50.19 \\
\hline & B & 0.536 & $\mathrm{n} / \mathrm{a}$ & 0.520 & 0.000 & $\mathrm{n} / \mathrm{a}$ & 0.445 \\
\hline \multirow{2}{*}{$V_{\mathrm{c}}\left[\mathrm{km} \mathrm{s}^{-1}\right]$} & A & 0.010 & $\mathrm{n} / \mathrm{a}$ & 0.100 & 0.851 & $\mathrm{n} / \mathrm{a}$ & 0.000 \\
\hline & B & -0.008 & $\mathrm{n} / \mathrm{a}$ & 0.000 & 0.600 & $\mathrm{n} / \mathrm{a}$ & 0.000 \\
\hline \multirow{2}{*}{$R_{l}[\mathrm{GV}]$} & A & 3.605 & 4.393 & $\mathrm{n} / \mathrm{a}$ & 4.651 & 4.603 & $\mathrm{n} / \mathrm{a}$ \\
\hline & B & -0.000 & 0.008 & n/a & 0.015 & 0.010 & $\mathrm{n} / \mathrm{a}$ \\
\hline \multirow{2}{*}{$\delta_{l}[-]$} & A & -0.525 & -0.696 & n/a & -0.803 & -0.784 & $\mathrm{n} / \mathrm{a}$ \\
\hline & B & -0.024 & -0.016 & $\mathrm{n} / \mathrm{a}$ & 0.025 & 0.007 & $\mathrm{n} / \mathrm{a}$ \\
\hline \multirow[t]{2}{*}{$\eta[-]$} & A & $\mathrm{n} / \mathrm{a}$ & $\mathrm{n} / \mathrm{a}$ & -0.140 & $\mathrm{n} / \mathrm{a}$ & $\mathrm{n} / \mathrm{a}$ & -1.713 \\
\hline & B & $\mathrm{n} / \mathrm{a}$ & $\mathrm{n} / \mathrm{a}$ & -0.208 & $\mathrm{n} / \mathrm{a}$ & $\mathrm{n} / \mathrm{a}$ & 0.116 \\
\hline
\end{tabular}

Notes. Two analyses are reported, based on the fit of AMS-02 B/C data only (left-hand side), or for the combined fit of AMS-02 Li/C, Be/B, and $\mathrm{B} / \mathrm{C}$ data (right-hand side).

Appendix $\mathrm{B}: L$ from several ${ }^{10} \mathrm{Be} /{ }^{9} \mathrm{Be}$ datasets

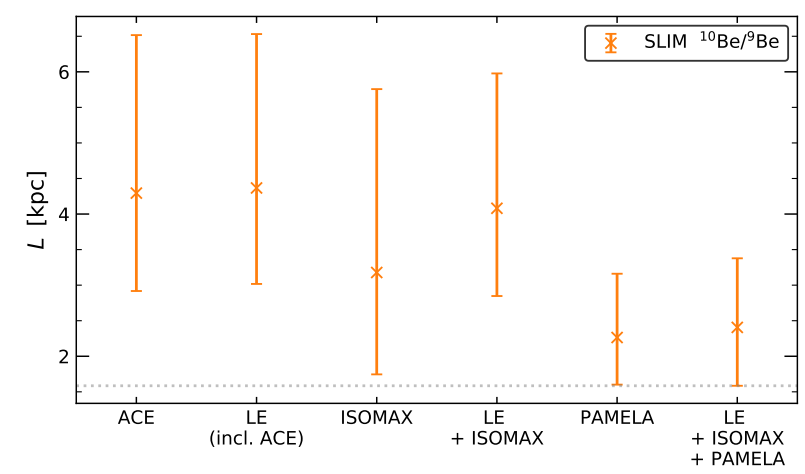

Fig. B.1. Constraints on $L$ (top panel) for combined fit of AMS-02 Li/C and $\mathrm{B} / \mathrm{C}$ data, and various datasets for ${ }^{10} \mathrm{Be} /{ }^{9} \mathrm{Be}$. From left to right: low-energy ACE data, all low-energy data combined (ACE, IMP7\&8, ISEE3, Ulysses-HET, and Voyager), higher-energy GeV/n ISOMAX (Hams et al. 2004), LE and ISOMAX combined (fourth column), preliminary PAMELA data (Bogomolov \& Vasilyev 2019), and all combined (last column).

The ${ }^{10} \mathrm{Be} /{ }^{9} \mathrm{Be}$ ratio has been measured by different experiments in different energy ranges. To better grasp the respective weight of the data in the final constraint (see Sect. 4), we show the different contributions to the fit. We focus on SLIM, but similar results are observed for BIG and QUAINT.

In Fig. B.1, we show the combined analysis of $\mathrm{Li} / \mathrm{C}, \mathrm{B} / \mathrm{C}$, and different datasets for ${ }^{10} \mathrm{Be} /{ }^{9} \mathrm{Be}$. Fits on low-energy data only are completely driven by ACE data (compare first and second column). They lead to larger $L$ values than the higher-energy ISOMAX data (third column). The latter have larger uncertainties than low-energy data (see Fig. 3), so that low-energy data drive the combined constraint (fourth column).

For completeness, we also show the constraints from the preliminary PAMELA data (Bogomolov \& Vasilyev 2019). They cover a energy range of 0.1 to $2 \mathrm{GeV} / \mathrm{n}$, combining two independent analyses with the TOF and calorimeter, with a $\sim 20 \%$ uncertainty. They give the best constraints on $L$ (next-to-last column) with smaller error bars, in line with the expectations discussed of Sect. 3.2. Most importantly, they point towards smaller values of the halo size than the other datasets, with $L \sim 2-3 \mathrm{pc}$. If all ${ }^{10} \mathrm{Be} /{ }^{9} \mathrm{Be}$ data are combined, the PAMELA data drive the fit (last column).
AMS-02 data will cover a similar range as PAMELA preliminary data. With probably slightly smaller uncertainties, they are expected to provide similar or slightly better precision on $L$. Because of the tension between PAMELA and lower-energy data, the results from both experiments are crucial to be able to obtain robust results on the central value of the halo size.

\section{Appendix C: Constraints from ${ }^{7} \mathrm{Be} /\left({ }^{9} \mathrm{Be}+{ }^{10} \mathrm{Be}\right)$}

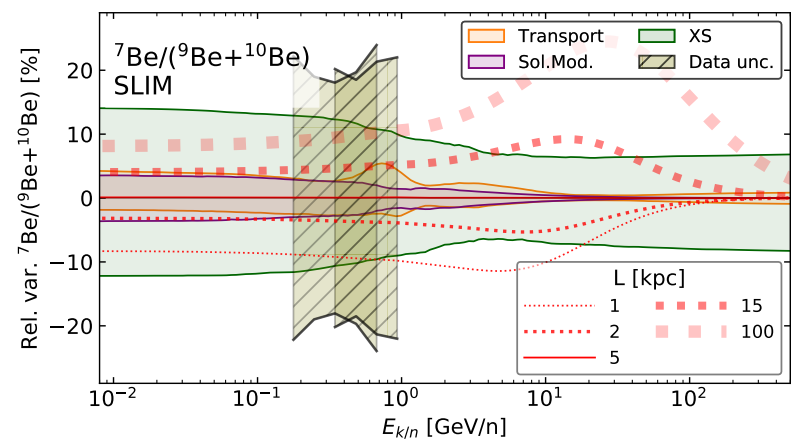

Fig. C.1. Same as Fig. 1, but for the variation of ${ }^{7} \mathrm{Be} /\left({ }^{9} \mathrm{Be}+{ }^{10} \mathrm{Be}\right)$ with $L$ (pinkish dashed lines), compared to various model uncertainties (contours) and current data uncertainties (hatched boxes); data from AMS01 (Aguilar et al. 2011) and PAMELA preliminary analysis (Menn et al. 2018).

Owing to the difficulty to achieve isotopic separation in CR experiments, data analyses often start with the most favourable configuration. The Be element is made of ${ }^{7} \mathrm{Be},{ }^{9} \mathrm{Be}$, and ${ }^{10} \mathrm{Be}$. Taking advantage of the $\Delta A=2$ mass separation between $A=7$ and $A=9,10$ isotopes, the ${ }^{7} \mathrm{Be} /\left({ }^{9} \mathrm{Be}+{ }^{10} \mathrm{Be}\right)$ ratio is experimentally a favourable configuration to analyse.

This ratio has been published by AMS-01 (Aguilar et al. 2011) and also by PAMELA (Menn et al. 2018). Following similar steps as the analysis presented in Sect. 3.2, we show in Fig. C.1 prospective limits on $L$ that can be set from using the ${ }^{7} \mathrm{Be} /\left({ }^{9} \mathrm{Be}+{ }^{10} \mathrm{Be}\right)$ ratio. Because of the sub-dominant abundance of ${ }^{10} \mathrm{Be}$ in the denominator, this ratio is as sensitive to $L$ as the $\mathrm{Be} / \mathrm{B}$ ratio. But whereas AMS-02 achieves a few percent precision on $\mathrm{Be} / \mathrm{B}$, current experiment are at $\sim 15-20 \%$ precision for isotopic ratios. For this reason, we conclude that ${ }^{7} \mathrm{Be} /\left({ }^{9} \mathrm{Be}+{ }^{10} \mathrm{Be}\right)$ is not a competitive target to fix $L$ with current data or with future experiments. 